\title{
Singularities of Tangent Varieties to Curves and Surfaces
}

\author{
Goo Ishikawa \\ Department of Mathematics, Hokkaido University, Japan \\ e-mail: ishikawa@math.sci.hokudai.ac.jp
}

\begin{abstract}
.
It is given the diffeomorphism classification on generic singularities of tangent varieties to curves with arbitrary codimension in a projective space. The generic classifications are performed in terms of certain geometric structures and differential systems on flag manifolds, via several techniques in differentiable algebra. It is provided also the generic diffeomorphism classification of singularities on tangent varieties to contact-integral curves in the standard contact projective space. Moreover we give basic results on the classification of singularities of tangent varieties to generic surfaces and Legendre surfaces.
\end{abstract}

\section{Introduction}

Embedded tangent spaces to a submanifold draw a variety in the ambient space, which is called the tangent variety to the submanifold. Tangent varieties appear in various geometric problems and applications naturally. See for instance [1] [9] [12]. Developable surfaces, varieties with degenerate Gauss mapping and varieties with degenerate projective dual are obtained by tangent varieties. Tangent varieties provide several important examples of non-isolated singularities in applications of geometry. We observe relations of tangent varieties to invariant theory and geometric theory of differential equations (see [29], also see Examples 2.7 and 9.1).

It is known, in the three dimensional Euclidean space, that the tangent variety (tangent developable) to a generic space curve has singularities each of which is locally diffeomorphic to the cuspidal edge or to the folded umbrella (cuspidal cross cap), as is found by Cayley and Cleave [8]. Cuspidal edge singularities appear along ordinary points, while the folded umbrella appears at an isolated point of zero torsion [12] 35].

The classification was generalised to more degenerate cases by Mond [32] 33] and Scherbak [38 [4] and applied to various geometry (see for instance [7] [24]). If we consider a curve together with its osculating framings, we are led to the classification of tangent varieties to generic osculating framed curves, possibly with singularities in themselves, in the three dimensional space. Then the list consists of 4 singularities: cuspidal edge, folded umbrella and moreover swallowtail and Mond surface ('cuspidal beak to beak') [21]. However the author could not find any literature treating the classification of singularities appearing in tangent varieties to higher codimensional curves.

The diffeomorphism types of tangent varieties to curves are invariant under projective transformations. In this paper, we consider curves in projective spaces and show the classification results on generic singularities of tangent varieties to curves with arbitrary codimension in projective spaces.

The tangent variety can be defined for a 'frontal' variety. A frontal variety has the well-defined embedded tangent space at each point, even where the variety is singular. In Cauchy problem of single unknown function, we have wave-front sets, which are singular hypersurfaces [3]. They are called fronts and form an important class of frontal varieties. Also higher codimensional wavefronts are examples of frontal varieties, which appear in, for instance, Cauchy problem of several 
unknown functions, where initial submanifolds of arbitrary codimension evolve to frontal varieties (cf. [13] 26]).

First, in $\$ 2$, we introduce the notion of frontal maps and frontal varieties, generalising that of submanifolds and fronts (Definition 2.1). Moreover we define their tangent maps and tangent varieties (Definition 2.2). Then we give the classification of tangent varieties to generic curves in projective spaces (Theorem 2.6). In fact we find that the tangent variety to a generic curve in $\mathbf{R} P^{N+1}$ has the unique singularity, the higher codimensional cuspidal edge, if $N+1 \geq 4$.

In the geometric theory of curves, however, we usually treat not just curves but we attach an appropriate frame with curves. Thus, to solve the generic classification problem properly, we relate the study of tangent varieties to certain kinds of differential systems on appropriate flag manifolds in \$3. Note that the method was initiated by Arnol'd and Scherbak [38. Also note that it is standard to use flag manifolds in the theory of space curves (40]). We can utilise various types of flag manifolds. In fact, in this paper, we select three kinds of flag manifolds and three kinds of differential systems, correspondingly to the classes of curves endowed with osculating-frames, with tangent-frames and with tangent-principal-normal-frames. Then we present the classification results on the singularities of which generically appear for these three kinds of classes of curves in projective spaces (Theorems 3.3, 3.4, 3.6. .

In $\$ 4$, the notion of types of curve-germs are recalled. Curves of finite type are frontal and their tangent varieties are frontal. We classify the generic types of curves, and then we show a kind of determinacy of the tangent variety for each generic type of curves.

In $\$ 5$, we classify the list of types of generic curves satisfying geometric conditions. To do this, we establish the codimension formulae giving the codimension of the set of curves, for given type, which satisfy a given geometric integrality condition in each case. Then the transversality theorem implies the restriction on types of generic curves.

In 86 , we introduce the key notion of openings of differentiable map-germs, which has close relations with that of frontal varieties. We collect necessary results on differentiable algebras to solve the generic classification problems treated in this paper. Moreover, in 87, using the method of differentiable algebra, we show the normal forms of tangent varieties appearing in the generic classification problems we have treated in this paper. In particular the main results in this paper, Theorems 2.6, 3.3, 3.4 and 3.6 are proved.

In 88 , we treat contact-integral curves and their tangent varieties. If $V$ is a symplectic vector space $V$, then the projective space $P(V)$ has the canonical contact structure. Then we give the generic diffeomorphism classification of singularities on tangent varieties to 'osculating framed contact-integral' curves in $P(V)$ (Theorems 8.5, 8.6). For this, in particular, we show that the diffeomorphism type of $\operatorname{Tan}(\gamma)$ is unique for a curve of type $(1,3,4,6)$ in $\mathbf{R} P^{4}$ in this paper. Note that it is known that the diffeomorphism type of $\operatorname{Tan} \operatorname{Tan}(\gamma)$ is not unique ([19]).

In $\$ 9$, we treat the classification problem of singularities of tangent varieties to surfaces, exhibiting several examples and observations. First we observe that the tangent varieties to a generic smooth surface are not frontal. We characterise the surface whose tangent variety is frontal. In particular we show that the tangent varieties to Legendre submanifolds in the five dimensional standard contact projective space $P\left(\mathbf{R}^{6}\right)=\mathbf{R} P^{5}$ are frontal, if the tangent variety has a dense regular set. Recall that the singularity of tangent variety to a curve along ordinary points is the cuspidal edge. Therefore the singularity of tangent variety at almost any point on a curve is diffeomorphic to cuspidal edge, which is a generic singularity of wave front. We study the analogous problem for tangent varieties to Legendre surfaces. Then we observe that the situation becomes absolutely different. In fact we introduce the notion of hyperbolic and elliptic ordinary points on Legendre surface in $\mathbf{R} P^{5}$ and show that the transverse section of the tangent variety to the surface, by a 3 -plane, has $D_{4}$-singularities (Theorem 9.5).

In the last section $\$ 10$, we collect open problems related to several results.

In this paper all manifolds and maps are assumed to be of class $C^{\infty}$ unless otherwise stated. 


\section{Frontal maps and tangent varieties.}

Definition 2.1 Let $N$ and $M$ be manifolds of dimension $n$ and $m$ respectively. Suppose $n \leq m$. A mapping $f: N^{n} \rightarrow M^{m}$ is called frontal if

(i) the regular locus

$$
\operatorname{Reg}(f)=\{x \in N \mid f:(N, x) \rightarrow(M, f(x)) \text { is an immersion }\}
$$

of $f$ is dense in $N$ and

(ii) there exists a $C^{\infty}$ mapping $\tilde{f}: N \rightarrow \operatorname{Gr}(n, T M)=\bigcup_{y \in M} \operatorname{Gr}\left(n, T_{y} M\right)$ satisfying

$$
\widetilde{f}(x)=f_{*}\left(T_{x} N\right), \quad \text { for } x \in \operatorname{Reg}(f) .
$$

Here $\operatorname{Gr}\left(n, T_{y} M\right)$ is the Grassmannian of $n$-planes in $T_{y} M$. Note that the lifting $\tilde{f}$ is uniquely determined if it exists and is called the Grassmannian lifting of $f$.

We define a subbundle $\mathcal{C} \subset T \operatorname{Gr}(n, T M)$ by setting, for $v \in T_{L} \operatorname{Gr}(n, T M), L \in T_{y} M$,

$$
v \in \mathcal{C}_{L} \Longleftrightarrow \pi_{*}(v) \in L \subset T_{y} M
$$

The differential system $\mathcal{C}$ is called the canonical differential system. The Grassmannian lifting $\tilde{f}$ is a $\mathcal{C}$-integral map, that is, $\widetilde{f}_{*}(T N) \subset \mathcal{C}$. We describe the canonical system in the next section Remark 3.7 in the case $M$ is a projective space.

If $f$ is an immersion, then $f$ is frontal. A wave-front hypersurface is frontal. The key observation for the classification of singularities of tangent varieties is that the tangent variety $\operatorname{Tan}(\gamma)$ to a curve $\gamma$ of finite type is frontal. the lifting Grassmannian is obtained by taking osculating planes to the curves (See \$4). If $n=m$, then $f$ is frontal if the condition (i) is fulfilled, $\widetilde{f}(x)$ being $T_{f(x)} \mathbf{R}^{m}$.

If $\tilde{f}$ is an immersion, then the frontal mapping is called a front. In [18], we called a frontal hypersurface $(m=\ell+1)$, a "front hypersurface". However we would like to reserve the notion "front" for the case that the Grassmannian lifting is an immersion, as in the Legendre singularity theory. Note that frontal maps are studied also in [28] [27] [36].

Definition 2.2 Let $f:\left(\mathbf{R}^{n}, a\right) \rightarrow\left(\mathbf{R}^{m}, b\right), n \leq m$ be a frontal map-germ and

$$
\widetilde{f}:\left(\mathbf{R}^{n}, a\right) \rightarrow \operatorname{Gr}\left(n, T \mathbf{R}^{m}\right) \cong \mathbf{R}^{m} \times \operatorname{Gr}\left(n, \mathbf{R}^{m}\right)
$$

be the Grassmannian lifting of $f$.

A tangent frame to $f$ means a system of vector fields $v_{1}, \ldots, v_{n}:\left(\mathbf{R}^{n}, a\right) \rightarrow T \mathbf{R}^{m}$ along $f$ such that $v_{1}(x), \ldots, v_{n}(x)$ form a basis of $\widetilde{f}(x) \subset T_{f(x)} \mathbf{R}^{m}$. Then the tangent map Tan $(f, v)$ : $\left(\mathbf{R}^{2 n},(a, 0)\right) \rightarrow\left(\mathbf{R}^{m}, b\right)$ is defined by

$$
\operatorname{Tan}(f, v)(s, x):=f(x)+\sum_{i=1}^{n} s_{i} v_{i}(x) .
$$

If we choose another tangent frame $u_{1}, \ldots, u_{n}$ of $f$ and define

$$
\operatorname{Tan}(f, u)(s, x)=f(x)+\sum_{i=1}^{n} s_{i} u_{i}(x) .
$$

Then $\operatorname{Tan}(f, u)$ and $\operatorname{Tan}(f, v)$ are right-equivalent. Therefore the tangent variety $\operatorname{Tan}(f)$ to a frontal map-germ is uniquely determined as a parametrised variety.

For a frontal map-germ $f:\left(\mathbf{R}^{n}, 0\right) \rightarrow \mathbf{R} P^{N+1}$ in a projective space we define the tangent map $\operatorname{Tan}(f):\left(\mathbf{R}^{n}, 0\right) \rightarrow \mathbf{R} P^{N+1}$ by taking a local projective coordinate $\left(\mathbf{R} P^{N+1}, f(0)\right) \rightarrow\left(\mathbf{R}^{N+1}, 0\right)$ (cf. 4 ). 
Remark 2.3 In this paper we treat only tangent varieties, which are closely related to the secant varieties. The secant variety of a submanifold $S \subset \mathbf{R} P^{n}$ is the ruled variety obtained by taking the union of secants connecting two distinct points on $S$ and by taking its closure ([41][11]). See also Example 9.1. The secant variety is parametrised by the 'secant map' and the tangent map is the 'boundary' of secant map in some sense. For the singularities of secant maps, see [14].

Let $\gamma:(\mathbf{R}, 0) \rightarrow \mathbf{R} P^{N+1}$ a germ of immersion and $\gamma(t)=\left(x_{1}(t), x_{2}(t), \ldots, x_{N+1}(t)\right)$ be a local representation of $\gamma$. Then $\gamma^{\prime}(t)$ gives the tangent frame of $\gamma$. Then the tangent variety to $\gamma$ is given by $\operatorname{Tan}(\gamma):\left(\mathbf{R}^{2}, 0\right) \rightarrow \mathbf{R}^{N+1}$ defined by

$$
\operatorname{Tan}(\gamma)(s, t)=\gamma(t)+s \gamma^{\prime}(t)=\left(x_{i}(t)+s x_{i}^{\prime}(t)\right)_{1 \leq i \leq N+1} .
$$

Note that $s$ is the parameter of tangent lines, while $t$ is the parameter of the original curve $\gamma$.

If $t=0$ is a singular point of $\gamma$, then the velocity vector $\gamma^{\prime}(0)=0$, and hence the above mapgerm does not give the parametrisation of the tangent variety. However if there is $k>0$ such that $v(t)=\left(1 / t^{k}\right) \gamma^{\prime}(t)$ is a tangent frame of $\gamma$, then we set

$$
\operatorname{Tan}(\gamma)(s, t)=\gamma(t)+s\left(\frac{1}{t^{k}} \gamma^{\prime}(t)\right)=\left(x_{i}(t)+s\left(\frac{1}{t^{k}} x_{i}^{\prime}(t)\right)\right)_{1 \leq i \leq N+1} .
$$

We take $k=0$ when $\gamma$ is an immersion at 0 .

In the above case, $\gamma$ is frontal and under a mild condition $\operatorname{Tan}(\gamma)$ is also frontal.

Theorem 2.4 Let $\gamma:(\mathbf{R}, 0) \rightarrow \mathbf{R} P^{N+1}$ be a curve of finite type (\$4). Then $\gamma$ is frontal. Moreover the tangent map $\operatorname{Tan}(\gamma):\left(\mathbf{R}^{2}, 0\right) \rightarrow \mathbf{R} P^{N+1}$ of $\gamma$ is frontal.

Theorem 2.4 is proved in $\$ 4$.

Remark 2.5 Let $\gamma$ be a curve of finite type. Then it is natural to ask what $\operatorname{Tan}(\operatorname{Tan}(\gamma))$ is, because $\operatorname{Tan}(\gamma)$ is frontal. For a curve $\gamma$ in $\mathbf{R} P^{N+1}, N \geq 2$, the tangent plane to $\operatorname{Tan}(\gamma)$ along each ruling (tangent line) is constant, that is the osculating 2-plane. Therefore $\operatorname{Tan}(\operatorname{Tan}(\gamma))$ is a 3 -fold, not a 4 -fold, ruled by osculating 2-planes of the original curve $\gamma([19])$.

We classify the map-germ $\operatorname{Tan}(\gamma)$ by local right-left diffeomorphism equivalence. Two mapgerms $f:(N, a) \rightarrow(M, b)$ and $f^{\prime}:\left(N^{\prime}, a^{\prime}\right) \rightarrow\left(M^{\prime}, b^{\prime}\right)$ are called diffeomorphic or right-left equivalent if there exist diffeomorphism-germs $\sigma:(N, a) \rightarrow\left(N^{\prime}, a^{\prime}\right)$ and $\tau:(M, b) \rightarrow\left(M^{\prime}, b^{\prime}\right)$ such that $f^{\prime} \circ \sigma=\tau \circ f$.

In the followings, $I$ is an open interval.

Theorem 2.6 (1) ([8]) For a generic curve $\gamma: I \rightarrow \mathbf{R} P^{3}$ in $C^{\infty}$-topology, the curve $\gamma$ is of finite type at each point in I and the tangent variety $\operatorname{Tan}(\gamma)$ to $\gamma$ at each point in I is locally diffeomorphic to the cuspidal edge or to the folded umbrella (cuspidal cross cap).

(2) Let $N+1 \geq 4$. For a generic curve $\gamma: I \rightarrow \mathbf{R} P^{N+1}$ in $C^{\infty}$-topology, the curve $\gamma$ is of finite type at each point in $I$ and the tangent variety $\operatorname{Tan}(\gamma)$ to $\gamma$ at each point of $I$ is locally diffeomorphic to the cuspidal edge.

The genericity means the existence of an open dense subset $\mathcal{O} \subset C^{\infty}\left(I, \mathbf{R} P^{N+1}\right)$ such that any $\gamma \in \mathcal{O}$ satisfies the consequence. by

The cuspidal edge is parametrised by the map-germ $\left(\mathbf{R}^{2}, 0\right) \rightarrow\left(\mathbf{R}^{N+1}, 0\right),(N+1 \geq 3)$ defined

$$
(u, x) \mapsto\left(u, x^{2}, x^{3}, 0, \ldots, 0\right) .
$$


Note that it is diffeomorphic (right-left equivalent) to the germ

$$
(t, s) \mapsto\left(t+s, t^{2}+2 s t, t^{3}+3 s t^{2}, \ldots, t^{N+1}+(N+1) s t^{N}\right),
$$

and also to

$$
(t, s) \mapsto\left(t+s, t^{2}+2 s t, t^{3}+3 s t^{2}, 0, \ldots, 0\right),
$$

A folded umbrella is parametrised by the germ $\left(\mathbf{R}^{2}, 0\right) \rightarrow\left(\mathbf{R}^{3}, 0\right)$ defined by

$$
(t, s) \mapsto\left(t+s, t^{2}+2 s t, t^{4}+4 s t^{3}\right),
$$

which is diffeomorphic to

$$
(u, x) \mapsto\left(u, x^{2}+u x, \frac{1}{2} x^{4}+\frac{1}{3} u x^{3}\right) .
$$

A folded umbrella is often called a cuspidal cross cap.
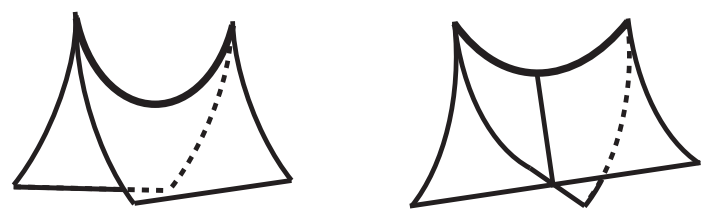

Figure 1: cuspidal edge and folded umbrella.

Theorem 2.6 is proved in $\$ 7$.

Example 2.7 (umbilical bracelet) Let

$$
V^{N+2}=\left\{a_{0} x^{N+1}+a_{1} x^{N} y+\cdots+a_{N} x y^{N}+a_{N+1} y^{N+1}\right\} \cong \mathbf{R}^{N+2}
$$

be the space of homogeneous polynomials of degree $N+1$ in two variables $x, y$. The polynomials with zeros of multiplicity $N+1$ form a curve $C$ in $P(V) \cong \mathbf{R} P^{N+1}$. The tangent variety $\operatorname{Tan}(C)$ to $C$ coincides with the set of polynomials with zeros of multiplicity $\geq N$. The surface $\operatorname{Tan}(C)$ has cuspidal edge singularities along $C$. In particular in the case $N+1=3$, the tangent variety $\operatorname{Tan}(C)$ to $C$ is called the umbilical bracelet([35] [10]). If $N+1 \geq 4, \operatorname{Tan}(\operatorname{Tan}(C)) \subset P\left(V^{N+2}\right)$ coincides with of polynomials with with zeros of multiplicity $\geq N-1$.

Remark 2.8 The tangent surface to a curve is obtained as a union of strata of envelope generated by the dual curve to the original curve. The generating family associated to the dual curve is determined, up to parametrised $\mathcal{K}$-equivalence in several cases. We recall the notion of types of curves in a projective space in $\$ 4$. If the type $\mathbf{A}=\left(a_{1}, \ldots, a_{N+1}\right)$ of a curve in $\mathbf{R} P^{N+1}$ is one of followings

$$
\begin{array}{ll}
(\mathrm{I})_{N, r} & :(1,2, \ldots, N, N+r),(r=0,1,2, \ldots), \\
(\mathrm{II})_{N, i} & :(1,2, \ldots, i, i+2, \ldots, N+1, N+2),(0 \leq i \leq N-1), \\
(\mathrm{III})_{N} & :(3,4, \ldots, N+2, N+3),
\end{array}
$$

then the generating family is determined by the type of the curve [17]. In each case, a normal form of the tangent variety can be obtained from the generating family

$$
F(t, x)=t^{a_{N+1}}+x_{1} t^{a_{N+1}-a_{1}}+x_{2} t^{a_{N+1}-a_{2}}+\cdots+x_{N} t^{a_{N+1}-a_{N}}+x_{N+1}=0,
$$

by solving

$$
F=0, \quad \frac{\partial F}{\partial t}=0, \quad \ldots, \quad \frac{\partial^{N-1} F}{\partial t^{N-1}}=0
$$


deleting the divisor $\{t=0\}$ if necessary. For example, for the type $(\mathrm{II})_{3,2}:(1,2,4,5)$, we have generating family

$$
F(t, x)=t^{5}+x_{1} t^{4}+x_{2} t^{3}+x_{3} t+x_{4} .
$$

Then the tangent variety is obtained by solving

$$
\left\{\begin{array}{l}
t^{5}+x_{1} t^{4}+x_{2} t^{3}+x_{3} t+x_{4}=0 \\
5 t^{4}+4 x_{1} t^{3}+3 x_{2} t^{2}+x_{3}=0 \\
20 t^{3}+12 x_{1} t^{2}+6 x_{2} t=0
\end{array}\right.
$$

In fact, from these equations, we get a map-germ $\left(\mathbf{R}^{2}, 0\right) \rightarrow\left(\mathbf{R}^{4}, 0\right)$ by

$$
x_{2}=-\frac{10}{3} t^{2}-2 x_{1} t, \quad x_{3}=5 t^{4}+2 x_{1} t^{3}, \quad x_{4}=-\frac{8}{3} t^{5}-x_{1} t^{4},
$$

which is diffeomorphic to the open folded umbrella (see Theorems 3.6, 7.2).

\section{Differential systems on flag manifolds.}

First we recall the flag manifolds and the canonical differential systems on flag manifolds. For the generality on differential systems, see [23].

Let $V$ be a vector space of dimension $n$ and $0<n_{1}<n_{2}<\cdots<n_{\ell}<n$. Then we define the flag manifold

$$
\mathcal{F}=\mathcal{F}_{n_{1}, n_{2}, \ldots, n_{\ell}}(V):=\left\{V_{n_{1}} \subset V_{n_{2}} \subset \cdots \subset V_{n_{\ell}} \subset V \mid \operatorname{dim}\left(V_{n_{j}}\right)=n_{j},(1 \leq j \leq \ell)\right\} .
$$

Note that

$$
\operatorname{dim}(\mathcal{F})=n_{1}\left(n-n_{1}\right)+\left(n_{2}-n_{1}\right)\left(n-n_{2}\right)+\cdots+\left(n_{\ell}-n_{\ell-1}\right)\left(n-n_{\ell}\right) .
$$

Denote by $\pi_{i}: \mathcal{F}_{n_{1}, n_{2}, \ldots, n_{\ell}}(V) \rightarrow \operatorname{Gr}\left(n_{i}, V\right)$ the canonical projection to the $i$-th member of the flag. The canonical differential system $\mathcal{C}=\mathcal{C}_{n_{1}, n_{2}, \ldots, n_{\ell}} \subset T \mathcal{F}$ is defined by, for $v \in T_{\mathbf{V}} \mathcal{F}, \mathbf{V} \in \mathcal{F}$,

$$
v \in \mathcal{C}_{\mathbf{V}} \Longleftrightarrow \pi_{i *}(v) \in T \operatorname{Gr}\left(n_{i}, V_{n_{i+1}}\right)\left(\subset T \operatorname{Gr}\left(n_{i}, V\right)\right),(1 \leq i \leq \ell-1) .
$$

Then $\mathcal{C}$ is a bracket-generating (completely non-integrable) subbundle of $T \mathcal{F}$ with

$$
\operatorname{rank}(\mathcal{C})=n_{1}\left(n_{2}-n_{1}\right)+\left(n_{2}-n_{1}\right)\left(n_{3}-n_{2}\right)+\cdots+\left(n_{\ell}-n_{\ell-1}\right)\left(n-n_{\ell}\right) .
$$

A $C^{\infty}$ curve $\Gamma: I \rightarrow \mathcal{F}$ from an open interval $I$ is called a $\mathcal{C}$-integral curve if $\Gamma^{\prime}(t) \in \mathcal{C}_{\Gamma(t)}$ for any $t \in I$. A $\mathcal{C}$-integral curve can be phrased as a $C^{\infty}$-family

$$
c(t)=\left(V_{n_{1}}(t), V_{n_{2}}(t), \ldots, V_{n_{\ell}}(t)\right)
$$

of flags in $\mathcal{F}$ such that each $V_{n_{i}}(t)$ moves along $V_{n_{i+1}}(t)$ at every moment infinitesimally.

Let $V$ be an $(N+2)$-dimensional vector space. For the study of tangent varieties to curves, it is natural to regard the following flag manifolds

$$
\mathcal{F}_{1,2}=\mathcal{F}_{1,2}(V):=\left\{V_{1} \subset V_{2} \subset V \mid \operatorname{dim}\left(V_{i}\right)=i\right\},
$$

and

$$
\mathcal{F}_{1,2,3}=\mathcal{F}_{1,2,3}(V):=\left\{V_{1} \subset V_{2} \subset V_{3} \subset V \mid \operatorname{dim}\left(V_{i}\right)=i\right\} .
$$

The canonical systems $\mathcal{T}=\mathcal{C}_{1,2}$ and $\mathcal{N}=\mathcal{C}_{1,2,3}$ are defined as follows: For $\left(V_{1}, V_{2}\right) \in \mathcal{F}_{1,2}$,

$$
v \in \mathcal{T}_{\left(V_{1}, V_{2}\right)} \Longleftrightarrow \pi_{1 *}(v) \in T P\left(V_{2}\right)(\subset T P(V)) .
$$

For $\left(V_{1}, V_{2}, V_{3}\right) \in \mathcal{F}_{1,2,3}$,

$$
w \in \mathcal{N}_{\left(V_{1}, V_{2}, V_{3}\right)} \Longleftrightarrow \pi_{1 *}(w) \in T P\left(V_{2}\right)(\subset T P(V)), \pi_{2 *}(w) \in T \operatorname{Gr}\left(2, V_{3}\right)(\subset T \operatorname{Gr}(2, V)) .
$$

Then we have 
Proposition 3.1 Let $\gamma:(\mathbf{R}, 0) \rightarrow P\left(V^{N+2}\right) \cong \mathbf{R} P^{N+1}$ be a $C^{\infty}$ curve. Suppose $\operatorname{Reg}(\gamma)$ is dense in $(\mathbf{R}, 0)$. Then $\gamma$ is frontal if and only if $\gamma=\pi_{1} \circ$ c for some $\mathcal{C}_{1,2}$-integral curve $c:(\mathbf{R}, 0) \rightarrow \mathcal{F}_{1,2}(V)$.

In fact $c$ gives a tangent frame of $\gamma$. In this case, $\gamma$ is called tangent-framed.

Proposition 3.2 Let $\gamma:(\mathbf{R}, 0) \rightarrow P\left(V^{N+2}\right) \cong \mathbf{R} P^{N+1}$ be a frontal curve. Suppose $\operatorname{Reg}(\operatorname{Tan}(\gamma))$ is dense in $\left(\mathbf{R}^{2}, 0\right)$. Then $\operatorname{Tan}(\gamma)$ is frontal if and only if $\gamma=\pi_{1} \circ \kappa$ for some $\mathcal{C}_{1,2,3}$-integral curve $\kappa:(\mathbf{R}, 0) \rightarrow \mathcal{F}_{1,2,3}(V)$.

In fact, if $\operatorname{Tan}(\gamma)$ is frontal, then $V_{1}(t)=\gamma(t)$, the tangent (projective) line $V_{2}(t)$ to $\gamma$ at $t$ and the tangent (projective) plane to $\operatorname{Tan}(\gamma)$ at $(t, 0)$ form a $\mathcal{C}_{1,2,3}$-integral lifting of $\gamma$. Conversely if $c(t)=\left(V_{1}(t), V_{2}(t), V_{3}(t)\right)$ is a $\mathcal{C}_{1,2,3}$-integral curve, then $\operatorname{Tan}(\gamma)$ has the constant tangent plane $V_{3}(t)$ along each ruling, and $(t, s) \mapsto T_{V_{1}(t)} P\left(V_{3}\right)(t)$ gives the Grassmannian lifting of Tan $(\gamma)$.

The projection of a $\mathcal{C}_{1,2,3}$-integral curve is called a tangent-principal-nomal-framed curve.

Theorem 3.3 (1) Let $N+1=3$. For a generic $\mathcal{C}_{1,2}$-integral curve $c: I \rightarrow \mathcal{F}_{1,2}\left(V^{4}\right)$ in $C^{\infty}$ topology, the tangent variety $\operatorname{Tan}(\gamma)$ to the tangent-framed curve $\gamma=\pi_{1} \circ c: I \rightarrow P\left(V^{4}\right)=\mathbf{R} P^{3}$ at each point is locally diffeomorphic to the cuspidal edge, the folded umbrella or the swallowtail.

(2) Let $N+1 \geq 4$. For a generic $\mathcal{C}_{1,2}$-integral curve $c: I \rightarrow \mathcal{F}_{1,2}\left(V^{N+2}\right)$ in $C^{\infty}$-topology, the tangent variety $\operatorname{Tan}(\gamma)$ to the tangent-framed curve $\gamma=\pi_{1} \circ c: I \rightarrow P(V)=\mathbf{R} P^{N+1}$ at each point is locally diffeomorphic to the cuspidal edge or the open swallowtail.

The swallowtail $\left(\mathbf{R}^{2}, 0\right) \rightarrow\left(\mathbf{R}^{3}, 0\right)$ is given by

$$
(t, s) \mapsto\left(t^{2}+2 s, t^{3}+3 s t, t^{4}+4 s t^{2}\right),
$$

which is diffeomorphic to

$$
(u, x) \mapsto\left(u, x^{3}+u x, \frac{3}{4} x^{4}+\frac{1}{2} u x^{2}\right) .
$$

The open swallowtail $\left(\mathbf{R}^{2}, 0\right) \rightarrow\left(\mathbf{R}^{N+1}, 0\right), N+1 \geq 4$ is given by

$$
(t, s) \mapsto\left(t^{2}+2 s, t^{3}+3 s t, t^{4}+4 s t^{2}, t^{5}+5 s t^{3}, 0, \ldots, 0\right)
$$

which is diffeomorphic to

$$
(u, x) \mapsto\left(u, x^{3}+u x, \frac{3}{4} x^{4}+\frac{1}{2} u x^{2}, \frac{3}{5} x^{5}+\frac{1}{3} u x^{3}, 0, \ldots, 0\right) .
$$

Theorem 3.4 (1) Let $N+1=3$. For a generic $\mathcal{C}_{1,2,3}$-integral curve $\kappa: I \rightarrow \mathcal{F}_{1,2,3}\left(V^{4}\right)$ in $C^{\infty}$ topology, the tangent variety $\operatorname{Tan}(\gamma)$ to the tangent-principal-normal-framed curve $\gamma=\pi_{1} \circ \kappa: I \rightarrow$ $P\left(V^{4}\right)=\mathbf{R} P^{3}$ at each point is locally diffeomorphic to the cuspidal edge, the folded umbrella, the Mond surface or the swallowtail.

(2) Let $N+1 \geq 4$. For a generic $\mathcal{C}_{1,2,3}$-integral curve $\kappa: I \rightarrow \mathcal{F}_{1,2,3}\left(V^{N+2}\right)$ in $C^{\infty}$-topology, the tangent variety $\operatorname{Tan}(\gamma)$ to the tangent-principal-normal-framed curve $\gamma=\pi_{1} \circ \kappa: I \rightarrow P(V)=$ $\mathbf{R} P^{N+1}$ at each point is locally diffeomorphic to the cuspidal edge, the open Mond surface or the open swallowtail.

The Mond surface $\left(\mathbf{R}^{2}, 0\right) \rightarrow\left(\mathbf{R}^{3}, 0\right)$ is given by

$$
\left(t+s, t^{3}+3 s t^{2}, t^{4}+4 s t^{3}\right),
$$

which is diffeomorphic to

$$
\left.(u, x) \mapsto\left(u, x^{3}+u x^{2}, \frac{3}{4} x^{4}+\frac{2}{3} u x^{3}\right)\right)
$$


The Mond surface is called also cuspidal beaks ([25]) or cuspidal beak to beak ('bec à bec').

The open Mond surface $\left(\mathbf{R}^{2}, 0\right) \rightarrow\left(\mathbf{R}^{N+1}, 0\right), N+1 \geq 4$ is given by

$$
\begin{gathered}
(t, s) \mapsto\left(t+s, t^{3}+3 s t^{2}, t^{4}+4 s t^{3}, t^{5}+5 s t^{4}, 0, \ldots, 0\right), \\
(u, x) \mapsto\left(u, x^{3}+u x^{2}, \frac{3}{4} x^{4}+\frac{2}{3} u x^{3}, \frac{3}{5} x^{5}+\frac{1}{2} u x^{4}, 0, \ldots, 0\right) .
\end{gathered}
$$

Now we recall on osculating-framed curves (cf. [21]). Let $V$ be an $(N+2)$-dimensional real vector space. Consider the complete flag manifold:

$$
\mathcal{F}=\mathcal{F}_{1,2, \ldots, N+1}(V):=\left\{V_{1} \subset V_{2} \subset \cdots V_{N+1} \subset V \mid \operatorname{dim}\left(V_{i}\right)=i, 1 \leq i \leq N+1\right\} .
$$

Then $\operatorname{dim} \mathcal{F}=\frac{(N+1)(N+2)}{2}$. We denote by $\pi_{i}: \mathcal{F} \rightarrow \operatorname{Gr}(i, V)$ the canonical projection

$$
\pi_{i}\left(V_{1}, V_{2}, \ldots, V_{N+1}\right)=V_{i} .
$$

The canonical system $\mathcal{C}=\mathcal{C}_{1,2, \ldots, N+1} \subset T \mathcal{F}$ is defined by

$$
v \in \mathcal{C}_{\left(V_{1}, \ldots, V_{N+1}\right)} \Longleftrightarrow \pi_{i *}(v) \in T \operatorname{Gr}\left(i, V_{i+1}\right)(\subset T \operatorname{Gr}(i, V)),(1 \leq i \leq N) .
$$

For a $C^{\infty}$ curve $\gamma: I \rightarrow P(V)=\mathbf{R} P^{N+1}$, if we consider Frenet-Serret frame, or the osculating projective moving frame, $\Gamma=\left(e_{0}(t), e_{1}(t), \ldots, e_{N+1}(t)\right): I \rightarrow \mathrm{GL}\left(\mathbf{R}^{N+2}\right)=\mathrm{GL}(N+2, \mathbf{R}), \gamma(t)=$ $\left[e_{0}(t)\right]$, then, setting $V_{i}(t):=\left\langle e_{0}(t), e_{1}(t), \ldots, e_{i-1}(t)\right\rangle_{\mathbf{R}},(1 \leq i \leq N+1)$, we have a $\mathcal{C}$-integral lifting $\widetilde{\gamma}: I \rightarrow \mathcal{F}$ of $\gamma$ for the projection $\pi_{1}: \mathcal{F} \rightarrow P(V)$, by $\widetilde{\gamma}(t)=\left(V_{1}(t), V_{2}(t), \ldots, V_{N+1}(t)\right)$. In this case, $\gamma$ is called osculating-framed. Note that the framing of an osculating-framed curve is uniquely determined if an orientation of the curve and a metric on $P(V)$ are given.

Theorem 3.5 ([21]) Let $N+1=3$. For a generic $\mathcal{C}$-integral curve $c: I \rightarrow \mathcal{F}\left(V^{4}\right)$ in $C^{\infty}$-topology, the tangent variety $\operatorname{Tan}(\gamma)$ to the osculating-framed curve $\gamma=\pi_{1} \circ c: I \rightarrow P\left(V^{4}\right)=\mathbf{R} P^{3}$ at each point of $I$ is locally diffeomorphic to the cuspidal edge, the folded umbrella, the swallowtail or to the Mond surface (Figure 2).
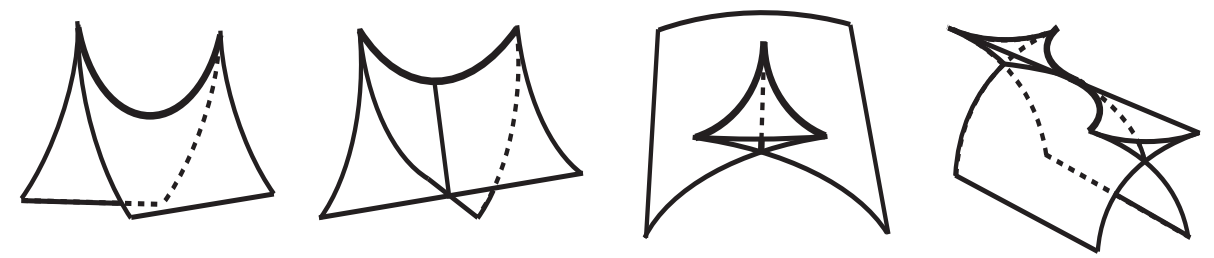

Figure 2: cuspidal edge, folded umbrella, swallowtail and Mond surface in $\mathbf{R}^{3}$.

In this paper we treat higher codimensional cases, and we show the following

Theorem 3.6 Let $N+1 \geq 4$. For a generic $\mathcal{C}$-integral curve $c: I \rightarrow \mathcal{F}$ in $C^{\infty}$-topology, the tangent variety to the osculating-framed curve $\gamma=\pi_{1} \circ c: I \rightarrow P\left(V^{N+2}\right)=\mathbf{R} P^{N+1}$ at each point is locally diffeomorphic to the cuspidal edge, the open folded umbrella (cuspidal non-cross cap), the open swallowtail or to the open Mond surface (Figure 3).

The open folded umbrella $\left(\mathbf{R}^{2}, 0\right) \rightarrow\left(\mathbf{R}^{N+1}, 0\right), N \geq 3$ is given by

$$
(t, s) \mapsto\left(t+s, t^{2}+2 s t, t^{4}+4 s t^{3}, t^{5}+5 s t^{4}, 0, \ldots, 0\right),
$$



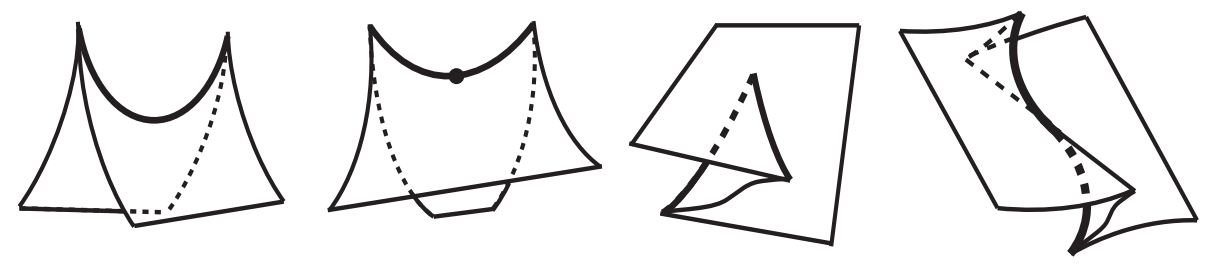

Figure 3: cuspidal edge, open folded umbrella, open swallowtail and open Mond surface in $\mathbf{R}^{4}$.

which is diffeomorphic to

$$
(u, x) \mapsto\left(u, x^{2}+u x, \frac{1}{2} x^{4}+\frac{1}{3} u x^{3}, \frac{2}{5} x^{5}+\frac{1}{4} u x^{4}, 0, \ldots, 0\right) .
$$

Our main results, Theorems $3.3,3.4,3.6$ are proved in $\$ 7$.

Lastly in this section, we describe the canonical system $\mathcal{C}=\mathcal{C}_{1,2, \ldots, k+1}$ on $\mathcal{F}_{1,2, \ldots, k+1}\left(V^{N+2}\right)$. Let $\mathbf{V}_{1}=\left(V_{11}, V_{21}, \ldots, V_{k+1,1}\right) \in \mathcal{F}_{1,2, \ldots, k+1}\left(V^{N+2}\right)$. Fix a flag $V^{N+2} \supset W_{N+1} \supset W_{N} \supset W_{N-k+1}$ such that $W_{N-i+1} \cap V_{i+1}=\{0\}, i=0,1, \ldots, k$. Take the open neighbourhood $U$ of $\mathbf{V}_{1}$ defined by

$$
U:=\left\{\left(V_{1}, V_{2}, \ldots, V_{k+1}\right) \in \mathcal{F}_{1,2, \ldots, k+1}\left(V^{N+2}\right) \quad \mid W_{N-i+1} \cap V_{i+1}=\{0\}, i=0,1, \ldots, k\right\} .
$$

Take non-zero vectors $e_{0} \in V_{11}, e_{1} \in V_{21} \cap W_{N+1}, e_{2} \in V_{31} \cap W_{N}, \ldots, e_{k} \in V_{k+11} \cap W_{N-k+2}$. Adding a basis $\left(e_{k+1}, \ldots, e_{N+1}\right)$ of $W_{N-k+1}$, we get a basis $\left(e_{0}, e_{1}, e_{2}, \ldots, e_{k}, e_{k+1}, \ldots, e_{N+1}\right)$ of $V$. Then, for each $\mathbf{V}=\left(V_{1}, V_{2}, \ldots, V_{k+1}\right), V_{i}$ has a basis $v_{0}, v_{1}, \ldots, v_{i-1}$ (a 'moving frame') of the form

$$
v_{i}=e_{i}+\sum_{j=i+1}^{N+1} x_{j}{ }^{i} e_{j}, \quad 0 \leq i \leq k .
$$

Then the condition that a curve in $\mathcal{F}_{1,2, \ldots, k+1}\left(V^{N+2}\right) \mathcal{C}$-integral is equivalent to that the components of the curve satisfies the conditions

$$
\left(v_{i-1}\right)^{\prime}=\sum_{j=i}^{N+1}\left(x_{j}{ }^{i-1}\right)^{\prime} e_{j} \in\left\langle v_{0}, v_{1}, \ldots, v_{i}\right\rangle_{\mathcal{E}_{1}}, \quad 1 \leq i \leq k .
$$

Thus we see that the differential system $\mathcal{C}=\mathcal{C}_{1,2, \ldots, k+1}$ is defined by

$$
d x_{j}^{i-1}-x_{j}{ }^{i} d x_{i}^{i-1}=0, \quad(1 \leq i \leq k, i+1 \leq j \leq N+1),
$$

for the system of local coordinates $\left(x_{j}{ }^{i}\right)_{0 \leq i \leq k, i+1 \leq j \leq N+1}$ of $\mathcal{F}_{1,2, \ldots, k+1}\left(V^{N+2}\right)$.

Remark 3.7 For a $(N+2)$-dimensional vector space $V$, the Grassmannian bundle $\operatorname{Gr}\left(n, T P\left(V^{N+2}\right)\right)$ over $P\left(V^{N+2}\right)$ is identified with the flag manifold $\mathcal{F}_{1, n+1}\left(V^{N+2}\right)$,

$$
\mathcal{F}_{1, n+1}\left(V^{N+2}\right)=\left\{V_{1} \subset V_{n+1} \subset V^{N+2} \mid \operatorname{dim}\left(V_{1}\right)=1, \operatorname{dim}\left(V_{n+1}\right)=n+1\right\} .
$$

Remark that the Grassmannian liftings of frontal maps $N^{n} \rightarrow P\left(V^{N+2}\right)$ are $\mathcal{C}$-integral of the canonical system $\mathcal{C}=\mathcal{C}_{1, n+1}$.

The canonical system $\mathcal{C}_{1, n+1}$ on $\mathcal{F}_{1, n+1}\left(V^{N+2}\right)$ is locally given by

$$
d x_{i+1}^{0}-\sum_{j=1}^{n} x_{i+1}^{j} d x_{j}^{0}=0, \quad(n \leq i \leq N)
$$


for a system of local coordinates $x_{i+1}^{0},(0 \leq i \leq N), x_{i+1}^{j},(1 \leq j \leq n, n \leq i \leq N)$. The projection $\pi_{1}: \operatorname{Gr}\left(n, T P\left(V^{N+2}\right)\right) \rightarrow P\left(V^{N+2}\right)$ is represented by $\left(x_{1}^{0}, \ldots, x_{N+1}^{0}\right)$. If we write $x_{i}=x_{i}^{0}(1 \leq i \leq$ $n), y_{k}=x_{n+k}^{0}(1 \leq k \leq N-n+1)$ and $p_{k}^{i}=x_{n+k}^{i}(1 \leq k \leq N-n+1,1 \leq i \leq n)$, then we have

$$
d y_{k}-\sum_{i=1}^{n} p_{k}^{i} d x_{i}=0, \quad 1 \leq k \leq N-n+1
$$

Therefore the condition that a map $F: L^{n} \rightarrow \operatorname{Gr}\left(n, T P\left(V^{N+2}\right)\right)$ is $\mathcal{C}$-integral is expressed by

$$
d\left(y_{k} \circ F\right)-\sum_{i=1}^{n}\left(p_{k}^{i} \circ F\right) d\left(x_{i} \circ F\right)=0, \quad 1 \leq k \leq N-n+1 .
$$

\section{Type of a curve in a space with flat projective structure.}

Let $M$ be an $m$-dimensional $C^{\infty}$ manifold. A flat projective structure on $M$ is given by an atlas $\left\{\left(U_{\alpha}, \varphi_{\alpha}\right)\right\}$ where $M=\bigcup_{\alpha} U_{\alpha}, \varphi_{\alpha}: U_{\alpha} \rightarrow \varphi_{\alpha}\left(U_{\alpha}\right) \subset \mathbf{R}^{m}$, and transition functions $\varphi_{\beta} \circ \varphi_{\alpha}^{-1}:$ $\varphi_{\alpha}\left(U_{\alpha} \cap U_{\beta}\right) \rightarrow \varphi_{\beta}\left(U_{\alpha} \cap U_{\beta}\right)$ are fractional linear with a common denominator. Then an admissible chart is called a system of projective local coordinates. The projective space $P\left(V^{m+1}\right)$ for a vector space $V^{m+1}$ has the canonical flat projective structure. Also Grassmannians and Lagrange Grassmannians have flat projective structures (cf. [15]).

Let $\gamma: I \rightarrow M$ be a $C^{\infty}$-curve in a manifold $M$ with a flat projective structure. Take a system of projective local coordinates $\left(x_{1}, x_{2}, \ldots, x_{m}\right)$ centred at $\gamma\left(t_{0}\right)$ and the local affine representation $\left(\mathbf{R}, t_{0}\right) \rightarrow\left(\mathbf{R}^{m}, 0\right)$,

$$
\gamma(t)={ }^{T}\left(x_{1}(t), x_{2}(t), \ldots, x_{m}(t)\right)
$$

of $\gamma$. Consider the $(m \times k)$-matrix

$$
W_{k}(t):=\left(\gamma^{\prime}\left(t_{0}\right), \gamma^{\prime \prime}\left(t_{0}\right), \cdots, \gamma^{(k)}\left(t_{0}\right)\right)
$$

for any integer $k \geq 1$ and $k=\infty$. Note that the rank of $W_{k}\left(t_{0}\right)$ is independent of the choice on representations for $\gamma$.

Definition 4.1 We call $\gamma$ of finite type at $t=t_{0} \in I$ if the $(m \times \infty)$-matrix

$$
W_{\infty}\left(t_{0}\right)=\left(\gamma^{\prime}\left(t_{0}\right), \gamma^{\prime \prime}\left(t_{0}\right), \cdots, \gamma^{(k)}\left(t_{0}\right), \cdots \cdots\right)
$$

is of rank $m$. Define, for $1 \leq i \leq m, a_{i}:=\min \left\{k \mid W_{k}\left(t_{0}\right)=i\right\}$. Then we have a sequence of natural numbers $1 \leq a_{1}<a_{2}<\cdots<a_{m}$, and we call $\gamma$ of type $\left(a_{1}, a_{2}, \ldots, a_{m}\right)$ at $t=t_{0} \in I$.

If $\left(a_{1}, a_{2}, \ldots, a_{m}\right)=(1,2, \ldots, m)$, then $t=t_{0}$ is called an ordinary point of $\gamma$.

It is easy to see

Lemma 4.2 A curve-germ $\gamma:(\mathbf{R}, 0) \rightarrow M$ in a manifold $M$ with a flat projective structure, is of type $\left(a_{1}, a_{2}, \ldots, a_{m}\right)$ at 0 if and only if there exists a system of projective local coordinates $\left(x_{1}, x_{2}, \ldots, x_{m}\right)$ centred at $\gamma(0)$ such that

$$
x_{1}(t)=t^{a_{1}}+o\left(t^{a_{1}}\right), x_{2}(t)=t^{a_{2}}+o\left(t^{a_{2}}\right), \ldots, x_{m}(t)=t^{a_{m}}+o\left(t^{a_{m}}\right) .
$$


Lemma 4.3 Let $\gamma:(\mathbf{R}, 0) \rightarrow P\left(\mathbf{R}^{N+2}\right)=\mathbf{R} P^{N+1}$ be a curve and $\widetilde{\gamma}:(\mathbf{R}, 0) \rightarrow \mathbf{R}^{N+2} \backslash\{0\}$ be a lifting of $\gamma$. Set $\widetilde{W}_{r}(t)=\left(\widetilde{\gamma}(t), \widetilde{\gamma}^{\prime}(t), \cdots, \widetilde{\gamma}^{(r)}(t)\right)$. Then $\gamma$ is of type $\mathbf{A}=\left(a_{1}, a_{2}, \ldots, a_{N+1}\right)$ if and only if $a_{i}=\min \left\{r \mid \widetilde{W}_{r}\left(t_{0}\right)=i+1\right\}, 1 \leq i \leq N+1$.

Moreover we see

Lemma 4.4 Let $\gamma:(\mathbf{R}, 0) \rightarrow P\left(\mathbf{R}^{N+2}\right)=\mathbf{R} P^{N+1}$ a curve of finite type. There is unique $\mathcal{C}_{1,2,3, \ldots, N+1}$-integral $C^{\infty}$ lifting $\Gamma:(\mathbf{R}, 0) \rightarrow \mathcal{F}_{1,2,3, \ldots, N+1}\left(\mathbf{R}^{N+2}\right)$ of $\gamma$. Moreover by the projection of $\Gamma$, we have $\mathcal{C}_{1,2,3}$-integral lifting $\kappa:(\mathbf{R}, 0) \rightarrow \mathcal{F}_{1,2,3}\left(\mathbf{R}^{N+1}\right)$ and $\mathcal{C}_{1,2}$-integral lifting $c:(\mathbf{R}, 0) \rightarrow \mathcal{F}_{1,2}\left(\mathbf{R}^{N+1}\right)$ of $\gamma$.

Proof: The first half is proved in [21] (Lemma 6.1). We take the lifting $\widetilde{\gamma}:(\mathbf{R}, 0) \rightarrow \mathbf{R}^{N+2} \backslash\{0\}$ defined by

$$
\widetilde{\gamma}(t)={ }^{T}\left(1, t^{a_{1}}+o\left(t^{a_{1}}\right), t^{a_{2}}+o\left(t^{a_{2}}\right), \ldots, t^{a_{N+1}}+o\left(t^{a_{N+1}}\right)\right) .
$$

of $\gamma$. Consider the $(N+2) \times(N+2)$-matrix

$$
A(t)=\left(\widetilde{\gamma}(t), \frac{1}{a_{1} !} \widetilde{\gamma}^{\left(a_{1}\right)}(t), \cdots, \frac{1}{a_{N+1} !} \widetilde{\gamma}^{\left(a_{N+1}\right)}(t)\right) .
$$

Let $V_{i}(t)$ be the linear subspace of $\mathbf{R}^{N+2}$ generated by the first $i$-columns of $A(t)$. Then $\Gamma$ : $(\mathbf{R}, 0) \rightarrow \mathcal{F}_{1,2,3, \ldots, N+1}\left(\mathbf{R}^{N+2}\right)$ is uniquely determined by $\Gamma(t)=\left(V_{1}(t), V_{2}(t), \ldots, V_{N+1}(t)\right)$. The lower triangle components of $A(t)$ give the local representation of $\Gamma$, therefore $\Gamma$ is $C^{\infty}$. Moreover $\kappa(t)=\left(V_{1}(t), V_{2}(t), V_{3}(t)\right)$ and $c(t)=\left(V_{1}(t), V_{2}(t)\right)$.

Proof of Theorem 2.4: Theorem 2.4 follows from Lemma 4.4 and Proposition 3.2 . Here we give concretely the Grassmannian lifting of $\operatorname{Tan}(\gamma)$ in term of Wronskian.

Lemma 4.5 Let $\gamma:(\mathbf{R}, 0) \rightarrow \mathbf{R} P^{N+1}$ be a curve-germ of type $\left(a_{1}, a_{2}, \ldots, a_{N+1}\right)$ and

$$
\gamma(t)=\left(x_{1}(t), x_{2}(t), \ldots, x_{N+1}(t)\right)
$$

be a local affine representation of $\gamma$. Then the tangent variety to $\gamma$ is parametrised by

$$
f(s, t)=\operatorname{Tan}(\gamma)(s, t):=\gamma(t)+s \frac{1}{\alpha(t)} \gamma^{\prime}(t)=\left(x_{i}(t)+s \frac{1}{\alpha(t)} x_{i}^{\prime}(t)\right)_{1 \leq i \leq N+1},
$$

where $\alpha(t)=t^{a_{1}-1}$. We set $f_{i}(s, t)=x_{i}(t)+\frac{s}{\alpha(t)} x_{i}^{\prime}(t)$. Then we have

$$
\frac{W_{i 2}}{W_{12}} d f_{1}+\frac{W_{1 i}}{W_{12}} d f_{2} .
$$

Here

$$
W_{i j}(t)=\left|\begin{array}{cc}
x_{i}^{\prime}(t) & x_{j}^{\prime}(t) \\
x_{i}^{\prime \prime}(t) & x_{j}^{\prime \prime}(t)
\end{array}\right|
$$

Proof: We have

$$
d f_{i}(s, t)=\frac{x_{i}^{\prime}(t)}{\alpha(t)} d s+\left(x_{i}^{\prime}(t)+s\left(\frac{x_{i}^{\prime}(t)}{\alpha(t)}\right)^{\prime}\right) d t
$$

Then we have

$$
\left|\begin{array}{cc}
x_{1}^{\prime} & x_{2}^{\prime} \\
x_{1}^{\prime}+s\left(\frac{x_{1}^{\prime}}{\alpha}\right)^{\prime} & x_{2}^{\prime}+s\left(\frac{x_{2}^{\prime}}{\alpha}\right)^{\prime}
\end{array}\right|=\frac{s}{\alpha} W_{12}
$$


Therefore we have, for $3 \leq i \leq n+1$,

$$
\begin{aligned}
& d f_{i}=\frac{\alpha}{s W_{12}}\left(d f_{1}, d f_{2}\right)\left(\begin{array}{cc}
x_{2}^{\prime}+s\left(\frac{x_{2}^{\prime}}{\alpha}\right)^{\prime} & -x_{2}^{\prime} \\
-x_{1}^{\prime}-s\left(\frac{x_{1}^{\prime}}{\alpha}\right)^{\prime} & x_{1}^{\prime}
\end{array}\right)\left(\begin{array}{c}
x_{i}^{\prime} \\
x_{i}^{\prime}+s\left(\frac{x_{i}^{\prime}}{\alpha}\right)^{\prime}
\end{array}\right) \\
& =\frac{W_{i 2}}{W_{12}} d f_{1}+\frac{W_{1 i}}{W_{12}} d f_{2} \text {. }
\end{aligned}
$$

Remark 4.6 Note that $\frac{W_{i 2}}{W_{12}}$ and $\frac{W_{1 i}}{W_{12}}$ are $C^{\infty}$ functions on $t$ of order $a_{i}-a_{1}, a_{i}-a_{2}$ respectively. The above formula gives the Grassmannian lifting $\tilde{f}:\left(\mathbf{R}^{2}, 0\right) \rightarrow \operatorname{Gr}\left(2, T \mathbf{R}^{N+1}\right)$ of $f=\operatorname{Tan}(\gamma)$.

Remark 4.7 If we set $g:\left(\mathbf{R}^{2}, 0\right) \rightarrow\left(\mathbf{R}^{2}, 0\right), g(s, t)=\left(f_{1}(s, t), f_{2}(s, t)\right)$. Then we have that $f_{3}, \ldots, f_{n+1} \in \mathcal{R}_{g}$ and that $f$ is an opening of $g$ in the sense of $\S 6$

\section{Codimension formulae and the genericity.}

We consider the jet space $J^{r}\left(I, \mathbf{R} P^{N+1}\right)$. Let $\mathbf{A}=\left(a_{1}, a_{2}, \ldots, a_{N}, a_{N+1}\right)$ be a strictly increasing sequence of positive integers. For $r>a_{N+1}$, we define

$$
\Sigma(\mathbf{A})=\left\{j^{r} \gamma\left(t_{0}\right) \mid t_{0} \in I, \gamma:\left(I, t_{0}\right) \rightarrow \mathbf{R} P^{N+1} \text { is of type } \mathbf{A} \text { at } t_{0}\right\} .
$$

Theorem $5.1([38]) \Sigma(\mathbf{A})$ is a semi-algebraic submanifold of codimension $\sum_{i=1}^{N+1}\left(a_{i}-i\right)$ in the jet space $J^{r}\left(I, \mathbf{R} P^{N+1}\right)$.

Proof: Let $J^{r}(1, N+1)$ be the fibre of the projection $\pi: J^{r}\left(I, \mathbf{R} P^{N+1}\right) \rightarrow I \times \mathbf{R} P^{N+1}$. Then $J^{r}(1, N+1)$ is identified with the space $\mathbf{R}^{(N+1) r}$ of $(N+1) \times r$-matrices. Then there exists an affine subspace $\Lambda \subset \mathbf{R}^{(N+1) r}$ such that $\Sigma(\mathbf{A})$ is an image of the polynomial embedding $\mathrm{GL}(N+1, \mathbf{R}) \times \Lambda \rightarrow$ $\mathbf{R}^{(N+1) r}$ defined by $(A, W) \mapsto A W$ for $A \in \mathrm{GL}(N+1, \mathbf{R}), W \in \Lambda$. Therefore $\Sigma(\mathbf{A})$ is a semialgebraic manifold.

The codimension of the set consisting of jets with $\operatorname{rank}\left(W_{a_{1}-1}\right)=0$ is equal to $(N+1)\left(a_{1}-\right.$ $1)$. The codimension of the set consisting of jets with $\operatorname{rank}\left(W_{a_{1}-1}\right)=0, \operatorname{rank}\left(W_{a_{1}}\right)=1$ and $\operatorname{rank}\left(W_{a_{2}-1}\right)=1$ is equal to $(N+1)\left(a_{1}-1\right)+N\left(a_{2}-a_{1}-1\right)$. Thus we have that the codimension of $\Sigma(\mathbf{A})$ is calculated as

$$
(N+1)\left(a_{1}-1\right)+N\left(a_{2}-a_{1}-1\right)+(N-1)\left(a_{3}-a_{2}-1\right)+\cdots+\left(a_{N+1}-a_{N}-1\right),
$$

which is equal to $\sum_{i=1}^{N+1}\left(a_{i}-i\right)$.

Corollary 5.2 For a generic curve $\gamma: I \rightarrow \mathbf{R} P^{N+1}$, and for any $t_{0} \in I$, the type of $\gamma$ at $t_{0}$ is equal to

$$
(1,2,3, \ldots, N, N+1) \text { or }(1,2,3, \ldots, N, N+2) .
$$

Proof: By the transversality theorem, there exists an open dense subset $\mathcal{O} \subset C^{\infty}\left(I, \mathbf{R} P^{N+1}\right)$ in $C^{\infty}$ topology such that for any $\gamma \in \mathcal{O}$ and for any $t_{0} \in I$, the type $\mathbf{A}$ of $\gamma$ at $t_{0}$ satisfies $\sum_{i=1}^{N+1}\left(a_{i}-i\right) \leq 1$. Then we have $a_{i}=i, 1 \leq i \leq N$ and $a_{N+1}=N+1$ or $a_{N+1}=N+2$, and thus we have the required result.

To treat osculating-framed curves, we consider the jet space of $\mathcal{C}$-integral curves, $\mathcal{C}=\mathcal{C}_{1,2, \ldots, N+1}$, $J_{\mathcal{C}}^{r}(I, \mathcal{F}) \subset J^{r}(I, \mathcal{F})$. Define

$$
\Sigma_{\mathcal{C}}(\mathbf{A}):=\left\{j^{r} \Gamma\left(t_{0}\right) \mid \Gamma:\left(\mathbf{R}, t_{0}\right) \rightarrow \mathcal{F} \text { is } \mathcal{C} \text {-integral, } \pi_{1} \circ \Gamma \text { is of type } \mathbf{A}\right\}
$$

in $J_{\mathcal{C}}^{r}(I, \mathcal{F})$ for sufficiently large $r$. 
Theorem $5.3([21]) \quad J_{\mathcal{C}}^{r}(I, \mathcal{F})$ is a submanifold of $J^{r}(I, \mathcal{F})$ and the codimension of $\Sigma_{\mathcal{C}}(\mathbf{A})$ in $J_{\mathcal{C}}^{r}(I, \mathcal{F})$ is equal to $a_{N+1}-(N+1)$.

Remark 5.4 Since any curve of finite type lifts to an $\mathcal{C}$-integral curve, $\Sigma_{\mathcal{C}}(\mathbf{A})$ is not empty for any $\mathbf{A}$.

By the transversality theorem for $\mathcal{C}$-integral curves, we have the following result:

Theorem 5.5 For a generic $\mathcal{C}$-integral curve $\Gamma: I \rightarrow \mathcal{F}_{1,2, \ldots, N+1}\left(V^{N+2}\right)$, the type $\mathbf{A}$ of $\pi_{1} \circ \Gamma$ at any point of $I$ is given by one of the following:

$$
\mathbf{A}=(1,2,3, \ldots, N, N+1), \quad(1,2, \ldots, i, i+2, \ldots, N+1, N+2),(i=0, \ldots, N) .
$$

Proof: By Theorem 5.3, for a genetic $\Gamma$, the type of $\pi_{1} \circ \Gamma$ at a point in $I$ satisfies that $a_{N+1}-$ $(N+1) \leq 1$, namely that $a_{N+1} \leq N+2$. Then we have the list of types.

In general, we consider the canonical system $\mathcal{C}=\mathcal{C}_{1,2, \ldots, k+1}$ on $\mathcal{F}=\mathcal{F}_{1,2, \ldots, k+1}\left(V^{N+2}\right)$, we consider the jet space of $\mathcal{C}$-integral curves, $J_{\mathcal{C}}^{r}(I, \mathcal{F}) \subset J^{r}(I, \mathcal{F})$. Define

$$
\Sigma_{\mathcal{C}}(\mathbf{A}):=\left\{j^{r} c\left(t_{0}\right) \mid c:\left(\mathbf{R}, t_{0}\right) \rightarrow \mathcal{F} \text { is } \mathcal{C} \text {-integral, } \pi_{1} \circ c \text { is of type } \mathbf{A}\right\}
$$

in $J_{\mathcal{C}}^{r}(I, \mathcal{F})$ for sufficiently large $r$.

Theorem 5.6 $J_{\mathcal{C}}^{r}(I, \mathcal{F})$ is a submanifold of $J^{r}(I, \mathcal{F})$ and the codimension of $\Sigma_{\mathcal{C}}(\mathbf{A})$ in $J_{\mathcal{C}}^{r}(I, \mathcal{F})$ is equal to

$$
\sum_{i=k}^{N+1}\left(a_{i}-i\right)-(N-k+1)\left(a_{k}-k\right)
$$

Note that, if $k=N$, the formula is reduced to $a_{N+1}-(N+1)$ (Theorem 5.3).

Proof of Theorem 5.6: Recall that $\mathcal{C}=\mathcal{C}_{1,2, \ldots, k+1}$ is defined by

$$
d x_{j}^{i-1}-x_{j}{ }^{i} d x_{i}{ }^{i-1}=0, \quad(1 \leq i \leq k, i+1 \leq j \leq N+1)
$$

for the system of local coordinates $\left(x_{j}{ }^{i}\right)_{0 \leq i \leq k, i+1 \leq j \leq N+1}$ of $\mathcal{F}_{1,2, \ldots, k+1}\left(V^{N+2}\right)($ \&3 . Then a $\mathcal{C}$ integral curve $\Gamma: I \rightarrow \mathcal{F}$ is obtained just form $x_{i}{ }^{i-1}$-components, $1 \leq i \leq k$, and $x_{j}{ }^{k}$-components, by integration. Then we see, at each point $t_{0} \in I, \operatorname{ord}\left(x_{j}^{0}=\sum_{\ell=1}^{j} \operatorname{ord}\left(x_{j}^{j-1}\right)\right.$. We have that the type of $\Gamma$ at $t_{0}$ is equal to $\mathbf{A}=\left(a_{1}, \ldots, a_{N+1}\right)$ if and only if

$$
\operatorname{ord}\left(x_{1}^{0}\right)=a_{1}, \operatorname{ord}\left(x_{2}^{1}\right)=a_{2}-a_{1}, \ldots, \operatorname{ord}\left(x_{k}^{k-1}\right)=a_{k}-a_{k-1},
$$

and the type of the curve $\left(x_{k+1}^{k}, \ldots, x_{N+1}^{k}\right):\left(I, t_{0}\right) \rightarrow \mathbf{R}^{N-k}$ is of type $\left(a_{k+1}-a_{k}, \ldots, a_{N+1}-a_{k}\right)$. Thus the codimension of $\Sigma_{\mathcal{C}}(\mathbf{A})$ is calculated as $\left(a_{1}-1\right)+\left(a_{2}-a_{1}-1\right)+\cdots+\left(a_{k}-a_{k-1}-1\right)+\sum_{k+1}^{N+1}\left(a_{j}-a_{k}-(j-k)\right)=\sum_{i=k}^{N+1}\left(a_{i}-i\right)-(N-k+1)\left(a_{k}-k\right)$. 
Remark 5.7 Let $\pi: \mathcal{F}_{1,2, \ldots, k, k+1} \rightarrow \mathcal{F}_{1,2, \ldots, k}$ be the canonical projection defined by

$$
\pi\left(V_{1}, V_{2}, \ldots, V_{k}, V_{k+1}\right)=\left(V_{1}, V_{2}, \ldots, V_{k}\right) .
$$

Then the $\pi$-fibres are projective subspaces of the flag manifold $\mathcal{F}_{1,2, \ldots, k+1}$. In the above proof, the functions $x_{k+1}^{k}, \ldots, x_{N+1}^{k}$ form a system of local projective coordinates of the $\pi$-fibre.

By the transversality theorem for $\mathcal{C}$-integral curves, we have the following results:

Theorem 5.8 For a generic $\mathcal{C}_{1,2}$-integral curve $c$, the type $\mathbf{A}$ of the tangent-framed curve $\pi_{1} \circ c$ at any point of $I$ is given by one of the following:

$$
(1,2,3, \ldots, N, N+1),(1,2,3, \ldots, N, N+2),(2,3,4, \ldots, N+1, N+2) .
$$

Proof: By Theorem 5.6, for a genetic $c$, the type of $\pi_{1} \circ c$ at a point in $I$ satisfies that $\sum_{i=1}^{N+1}\left(a_{i}-i\right)-$ $N\left(a_{1}-1\right) \leq 1$, namely that $\sum_{i=1}^{N+1}\left(a_{i}-i\right) \leq N\left(a_{1}-1\right)+1$. Then $(N+1)\left(a_{1}-1\right) \leq \sum_{i=1}^{N+1}\left(a_{i}-i\right) \leq$ $N\left(a_{1}-1\right)+1$. Therefore $a_{1} \leq 2$ and, if $a_{1}=2$, then $\mathbf{A}=(2,3,4, \ldots, N+1, N+2)$. If $a_{1}=1$, then $\sum_{i=1}^{N+1}\left(a_{i}-i\right) \leq 1$. Therefore we have the result.

Theorem 5.9 For a generic $\mathcal{C}_{1,2,3}$-integral curve $\kappa$, the type $\mathbf{A}$ of the tangent-principal-normalframed curve $\pi_{1} \circ \kappa$ at any point of $I$ is given by one of the following:

$$
(1,2,3, \ldots, N, N+1),(1,2,3, \ldots, N, N+2),(1,3,4, \ldots, N+1, N+2),(2,3,4, \ldots, N+1, N+2) .
$$

Proof: By Theorem 5.6 for a genetic $c$, the type of $\pi_{1} \circ c$ at a point in $I$ satisfies that $\sum_{i=2}^{N+1}\left(a_{i}-\right.$ $i)-(N-1)\left(a_{2}-2\right) \leq 1$, namely that $\sum_{i=2}^{N+1}\left(a_{i}-i\right) \leq(N-1)\left(a_{2}-2\right)+1$. Then $N\left(a_{2}-2\right) \leq$ $\sum_{i=2}^{N+1}\left(a_{i}-i\right) \leq(N-1)\left(a_{2}-2\right)+1$, and we have $a_{2} \leq 3$. If $a_{2}=3$, then $\mathbf{A}=(1,3,4, \ldots, N+1, N+2)$ or $(2,3,4, \ldots, N+1, N+2)$. If $a_{2}=2$, then $\mathbf{A}=(1,2,3, \ldots, N, N+1)$ or $(1,2,3, \ldots, N, N+2)$.

Remark 5.10 We observe that, in all lists of the generic classifications of types, there are just three possibilities of the leading two digits: $(1,2),(1,3)$ and $(2,3)$. These cases correspond to the cases where the projection of the tangent variety to the osculating plane is diffeomorphic to the map-germ $\left(\mathbf{R}^{2}, 0\right) \rightarrow\left(\mathbf{R}^{2}, 0\right)$, the fold singularities $(x, u) \mapsto\left(x^{2}, u\right)$, 'beak to beak' $(x, u) \mapsto\left(x^{3}+u x^{2}, u\right)$ and Whitney's cusp map $(x, u) \mapsto\left(x^{3}+u x, u\right)$ respectively.

\section{Opening procedure of differentiable map-germs.}

To describe singularities of frontal mappings, we introduce the notion of "openings" of mappings.

The tangent variety to a curve in $\mathbf{R} P^{N+1}$ projects locally to the tangent variety to a space curve in the osculating 3-space, and to a plane curve in the osculating 2-plane. Then the tangent variety in $\mathbf{R} P^{N+1}$ can be regarded as an "opening" of a tangent variety to a space curve and to a plane curve. For example, the open swallowtail, which is an opening of the swallowtail, appears in many context. It appears as a singular Lagrangian variety [2], and as a singular solution to certain partial differential equation [13. The open folded umbrella appears as a 'frontal-symplectic singularity' ([22]).

We denote by $\mathcal{E}_{a}$ the $\mathbf{R}$-algebra of $C^{\infty}$ function-germs on $\left(\mathbf{R}^{n}, a\right)$ with the maximal ideal $\mathfrak{m}_{a}$. If $a$ is the origin, then we use $\mathcal{E}_{n}, \mathfrak{m}_{n}$ instead of $\mathcal{E}_{a}, \mathfrak{m}_{a}$ respectively. 


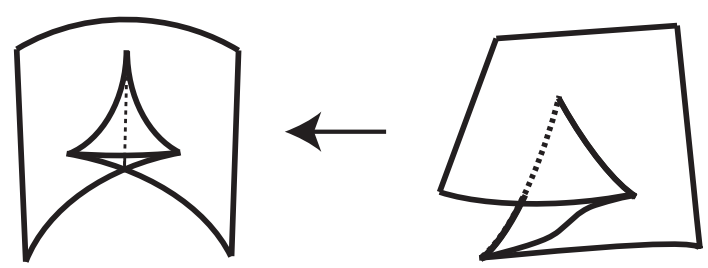

Figure 4: Opening of swallowtail.

Definition 6.1 ([16] [20]) Let $f:\left(\mathbf{R}^{n}, a\right) \rightarrow\left(\mathbf{R}^{m}, b\right)$ be a $C^{\infty}$ map-germ with $n \leq m$. We define the Jacobi module of $f$ :

$$
\mathcal{J}_{f}=\left\{\sum_{j=1}^{m} p_{j} d f_{j} \mid a_{j} \in \mathcal{E}_{a},(1 \leq j \leq m)\right\} \subset \Omega_{a}^{1},
$$

in the space $\Omega_{a}^{1}$ of 1 -form germs on $\left(\mathbf{R}^{n}, a\right)$. Further we define the ramification module $\mathcal{R}_{f}$ by

$$
\mathcal{R}_{f}:=\left\{h \in \mathcal{E}_{a} \mid d h \in \mathcal{J}_{f}\right\}
$$

Note that $\mathcal{J}_{f}$ is just the first order component of the graded differential ideal $\mathcal{J}_{f}^{\bullet}$ in $\Omega_{a}^{\bullet}$ generated by $d f_{1}, \ldots, d f_{m}$. Then the singular locus is given by $\Sigma_{f}=\left\{x \in\left(\mathbf{R}^{n}, a\right) \mid \operatorname{rank} \mathcal{J}_{f}(x)<n\right\}$. Also we consider the Kernel field $\operatorname{Ker}\left(f_{*}: T \mathbf{R}^{n} \rightarrow T \mathbf{R}^{m}\right)$, of $f$ near $a$. Then we see that, for another mapgerm $f^{\prime}:\left(\mathbf{R}^{n}, a\right) \rightarrow\left(\mathbf{R}^{m^{\prime}}, b^{\prime}\right)$ with $\mathcal{J}_{f^{\prime}}=\mathcal{J}_{f}, n \leq m^{\prime}$, we have $\Sigma_{f^{\prime}}=\Sigma_{f}$ and $\operatorname{Ker}\left(f_{*}^{\prime}\right)=\operatorname{Ker}\left(f_{*}\right)$.

Related notion was introduced in [34].

Lemma 6.2 Let $f:\left(\mathbf{R}^{n}, a\right) \rightarrow\left(\mathbf{R}^{m}, b\right)$ be a $C^{\infty}$ map-germ.

(1) $f^{*} \mathcal{E}_{b} \subset \mathcal{R}_{f} \subset \mathcal{E}_{a}$ and $\mathcal{R}_{f}$ is an $\mathcal{E}_{b}$-module via $f^{*}$.

(2) For another map-germ $f^{\prime}:\left(\mathbf{R}^{n}, a\right) \rightarrow\left(\mathbf{R}^{m^{\prime}}, b^{\prime}\right), \mathcal{J}_{f^{\prime}}=\mathcal{J}_{f}$ if and only if $\mathcal{R}_{f^{\prime}}=\mathcal{R}_{f}$.

(3) If $\tau:\left(\mathbf{R}^{m}, b\right) \rightarrow\left(\mathbf{R}^{m}, b^{\prime}\right)$ is a diffeomorphism-germ, then $\mathcal{R}_{\tau \circ f}=\mathcal{R}_{f}$. If $\sigma:\left(\mathbf{R}^{n}, a^{\prime}\right) \rightarrow$ $\left(\mathbf{R}^{n}\right.$, a) is a diffeomorphism-germ, then $\mathcal{R}_{f \circ \sigma}=\sigma^{*}\left(\mathcal{R}_{f}\right)$.

Proof: (1) follows from that, if $h \in \mathcal{R}_{f}$ and $d h=\sum_{j=1}^{m} p_{j} d f_{j}$, then we have

$$
d\{(k \circ f) h\}=\sum_{j=1}^{m}\left\{(k \circ f) p_{j}+h\left(\partial k / \partial y_{j}\right)\right\} d f_{j} .
$$

(2) It is clear that $\mathcal{J}_{f^{\prime}}=\mathcal{J}_{f}$ implies $\mathcal{R}_{f^{\prime}}=\mathcal{R}_{f}$. Conversely suppose $\mathcal{R}_{f^{\prime}}=\mathcal{R}_{f}$. Then any component $f_{j}^{\prime}$ of $f^{\prime}$ belongs to $\mathcal{R}_{f^{\prime}}=\mathcal{R}_{f}$, hence $d f_{j} \in \mathcal{J}_{f}$. Therefore $\mathcal{J}_{f^{\prime}} \subset \mathcal{J}_{f}$. By the symmetry we have $\mathcal{J}_{f^{\prime}}=\mathcal{J}_{f}$.

(3) follows from that $\mathcal{J}_{\tau \circ f}=\mathcal{J}_{f}$ and $\mathcal{J}_{f \circ \sigma}=\sigma^{*}\left(\mathcal{J}_{f}\right)$.

Definition 6.3 Let $f:\left(\mathbf{R}^{n}, a\right) \rightarrow\left(\mathbf{R}^{m}, b\right), n \leq m$ be a $C^{\infty}$ map-germ. Given $h_{1}, \ldots, h_{r} \in \mathcal{R}_{f}$, the map-germ $F:\left(\mathbf{R}^{n}, 0\right) \rightarrow \mathbf{R}^{m} \times \mathbf{R}^{r}=\mathbf{R}^{m+r}$ defined by

$$
f=\left(f_{1}, \ldots, f_{m}, h_{1}, \ldots, h_{r}\right)
$$

is called an opening of $f$, while $f$ is called a closing of $F$.

An opening $F=\left(f, h_{1}, \ldots, h_{r}\right)$ of $f$ is called a versal opening (resp. mini-versal opening) of $f$, if $1, h_{1}, \ldots, h_{r}$ form a (minimal) system of generators of $\mathcal{R}_{f}$ as an $\mathcal{E}_{b^{-}}$-module via $f^{*}$. 
Note that an opening of an opening of $f$ is an opening of $f$.

Here we summarise known results on the ramification module. A map-germ $f:\left(\mathbf{R}^{n}, a\right) \rightarrow$ $\left(\mathbf{R}^{m}, b\right)$ is called finite if $\operatorname{dim}_{\mathbf{R}} \mathcal{E}_{a} /\left(f^{*} \mathfrak{m}_{b}\right) \mathcal{E}_{a}<\infty$.

Proposition 6.4 (Theorem 3.1 of [18], Corollary 2.4 of [20]) If $f:\left(\mathbf{R}^{n}, a\right) \rightarrow\left(\mathbf{R}^{m}, b\right)$ is finite and of corank at most one. Then we have

(1) $\mathcal{R}_{f}$ is a finite $\mathcal{E}_{b}$-module. Therefore there exists a versal opening of $f$.

(2) $1, h_{1}, \ldots, h_{r} \in \mathcal{R}_{f}$ generate $\mathcal{R}_{f}$ as $\mathcal{E}_{b}$-module if and only if they generate the vector space $\mathcal{R}_{f} / f^{*}\left(\mathfrak{m}_{b}\right) \mathcal{R}_{f}$ over $\mathbf{R}$.

Remark 6.5 By Proposition 6.4, we see that $1, h_{1}, \ldots, h_{r} \in \mathcal{R}_{f}$ form a minimal system of generators of $\mathcal{R}_{f}$ as $\mathcal{E}_{b}$-module if and only if they form a basis of $\mathbf{R}$-vector space $\mathcal{R}_{f} / f^{*}\left(\mathfrak{m}_{b}\right) \mathcal{R}_{f}$.

Let $k \geq 0, m \geq 0$. To present the normal forms of Morn map, consider variables $t, \lambda=$ $\left(\lambda_{1}, \ldots, \lambda_{k-1}\right), \mu=\left(\mu_{i j}\right)_{1 \leq i \leq m, 1 \leq j \leq k}$ and polynomials

$$
F(t, \lambda)=t^{k+1}+\sum_{i=1}^{k-1} \lambda_{j} t^{j}, \quad G_{i}(t, \mu)=\sum_{j=1}^{k} \mu_{i j} t^{j},(1 \leq i \leq m) .
$$

Let $f:\left(\mathbf{R}^{k+k m}, 0\right) \rightarrow\left(\mathbf{R}^{m+k+k m}, 0\right)$ be a Morin map defined by

$$
f(t, \lambda, \mu):=(F(t, \lambda), G(t, \mu), \lambda, \mu),
$$

for the above polynomials $F$ and $G$.

For $\ell \geq 0$, we denote by $F_{(\ell)}, G_{i(\ell)}$ the polynomials

$$
F_{(\ell)}(t, \lambda)=\int_{0}^{t} s^{\ell} F(s, \lambda) d s, \quad G_{i(\ell)}(t, \mu)=\int_{0}^{t} s^{\ell} G_{i}(s, \mu) d s .
$$

Then we have:

Proposition 6.6 (Theorem 3 of [16]) The ramification module $\mathcal{R}_{f}$ of the Morin map $f$ is minimally generated over $f^{*} \mathcal{E}_{m+k+k m}$ by the $1+k+(k-1) m$ elements

$$
1, F_{(1)}, \ldots, F_{(k)}, G_{1(1)}, \ldots, G_{1(k-1)}, \ldots, G_{m(1)}, \ldots, G_{m(k-1)} .
$$

The map-germ $\mathbf{F}:\left(\mathbf{R}^{k+m k}, 0\right) \rightarrow\left(\mathbf{R}^{m+k+k m} \times \mathbf{R}^{k+(k-1) m}, 0\right)=\left(\mathbf{R}^{2(k+k m)}, 0\right)$ defined by

$$
\mathbf{F}=\left(f, F_{(1)}, \ldots, F_{(k)}, G_{1(1)}, \ldots, G_{1(k-1)}, \ldots, G_{m(1)}, \ldots, G_{m(k-1)}\right)
$$

is a versal opening of $f$.

Remark 6.7 It is shown in [16] moreover that $\mathbf{F}$ is an isotropic map for a symplectic structure on $\mathbf{R}^{2(k+k m)}$.

Proposition 6.8 (cf. Proposition 1.6 of [18, Lemma 2.4 of [19]) Let $f:\left(\mathbf{R}^{n}, a\right) \rightarrow\left(\mathbf{R}^{m}, b\right)$ be $a$ $C^{\infty}$ map-germ and $F:\left(\mathbf{R}^{n+\ell},(a, 0)\right) \rightarrow\left(\mathbf{R}^{m+\ell},(b, 0)\right)$ be an unfolding of $f: F(x, u)=\left(F_{1}(x, u), u\right)$ and $F_{1}(x, 0)=f(x)$. Let $i:\left(\mathbf{R}^{n}, a\right) \rightarrow\left(\mathbf{R}^{n+\ell},(a, 0)\right)$ be the inclusion, $i(x)=(x, 0)$. Then we have:

(1) $i^{*} \mathcal{R}_{F} \subset \mathcal{R}_{f}$.

(2) If $f$ is of corank $\leq 1$ with $n \leq m$, then $i^{*} \mathcal{R}_{F}=\mathcal{R}_{f}$. If $1, H_{1}, \ldots, H_{r}$ generate $\mathcal{R}_{F}$ via $F^{*}$, then $1, i^{*} H_{1}, \ldots, i^{*} H_{r}$ generate $\mathcal{R}_{f}$ via $f^{*}$.

(3) Let $\ell$ be a positive integer and $F=\left(F_{1}(t, u), u\right):\left(\mathbf{R}^{n}, 0\right) \rightarrow\left(\mathbf{R}^{n}, 0\right)$ an unfolding of $f$ : $(\mathbf{R}, 0) \rightarrow(\mathbf{R}, 0), f(t)=F_{1}(t, 0)=t^{\ell}$. Suppose $H_{1}, \ldots, H_{r} \in \mathcal{R}_{F} \cap \mathfrak{m}_{n}$. Then $1, H_{1}, \ldots, H_{r}$ generate $\mathcal{R}_{F}$ via $F^{*}$ if and only $i^{*} H_{1}, \ldots, i^{*} H_{r}$ generate $\mathfrak{m}_{1}^{\ell+1} / \mathfrak{m}_{1}^{2 \ell}$. In particular $F_{1(1)}, \ldots, F_{1(\ell-1)}$ form a system of generators of $\mathcal{R}_{F}$ via $F^{*}$ over $\mathcal{E}_{n}$. 
Proof: (1) is clear. (2) Let $H \in \mathcal{R}_{F}$. Then $d H \in \mathcal{J}_{F}$. Hence $d\left(i^{*} H\right)=i^{*}(d H) \in i^{*} \mathcal{J}_{F} \subset$ $\mathcal{J}_{f}$. Therefore $i^{*} H \in \mathcal{R}_{f}$. Let $f$ be of corank at most one. Suppose $h \in \mathcal{R}_{f}$. Then $d h=$ $\sum_{j=1}^{m} a_{j} d f_{j}$ for some $a_{j} \in \mathcal{E}_{a}$. There exist $A_{j}, B_{k} \in \mathcal{E}_{(a, 0)}$ such that $i^{*} A_{j}=a_{j}$ and the 1-form $\sum_{j=1}^{m} A_{j} d\left(F_{1}\right)_{j}+\sum_{k=1}^{\ell} B_{k} d \lambda_{k}$ is closed (cf. Lemma 2.5 of [20]). Then there exists an $H \in \mathcal{E}_{(a, 0)}$ such that $d H=\sum_{j=1}^{m} A_{j} d\left(F_{1}\right)_{j}+\sum_{k=1}^{\ell} B_{k} d \lambda_{k} \in \mathcal{J}_{F}$ and $d\left(i^{*} H\right)=i^{*}(d H)=d h$. Then there exists $c \in \mathbf{R}$ such that $h=i^{*} H+c=i^{*}(H+c)$, and $H+c \in \mathcal{R}_{F}$. Therefore $h \in i^{*} \mathcal{R}_{F}$. Since $i^{*}$ is a homomorphism over $j^{*}: \mathcal{E}_{(b, 0)} \rightarrow \mathcal{E}_{b}$, where $j:\left(\mathbf{R}^{m}, 0\right) \rightarrow\left(\mathbf{R}^{m+\ell}, 0\right)$ is the inclusion $j(y)=(y, 0)$, we have the consequence. (3) It is easy to show that $\mathcal{R}_{f}=\mathbf{R}+\mathfrak{m}_{1}^{\ell}$. By Proposition 6.4 (2), $1, H_{1}, \ldots, H_{r}$ generate $\mathcal{R}_{F}$ as $\mathcal{E}_{n}$-module via $F^{*}$ if and only if they generate $\mathcal{R}_{F} / F^{*}\left(\mathfrak{m}_{n}\right) \mathcal{R}_{F}$ over R. Since

$$
\mathcal{R}_{F} / F^{*}\left(\mathfrak{m}_{n}\right) \mathcal{R}_{F} \cong\left(\mathbf{R}+\mathfrak{m}_{1}^{\ell}\right) /\left(f^{*} \mathfrak{m}_{1}\right)\left(\mathbf{R}+\mathfrak{m}_{1}^{\ell}\right) \cong \mathfrak{m}_{1}^{\ell+1} / \mathfrak{m}_{1}^{2 \ell}
$$

we have the consequence.

Proposition 6.9 Let $f:\left(\mathbf{R}^{n}, a\right) \rightarrow\left(\mathbf{R}^{m}, b\right), n \leq m$ be a $C^{\infty}$ map-germ.

(1) For any versal opening $F:\left(\mathbf{R}^{n}, a\right) \rightarrow\left(\mathbf{R}^{m+r}, F(a)\right)$ of $f$ and for any opening $G:\left(\mathbf{R}^{n}, a\right) \rightarrow$ $\left(\mathbf{R}^{m+s}, G(a)\right)$, there exists an affine bundle map $\Psi:\left(\mathbf{R}^{m+r}, F(a)\right) \rightarrow\left(\mathbf{R}^{m+s}, G(a)\right)$ over $\left(\mathbf{R}^{m}, f(a)\right)$ such that $G=\Psi \circ F$.

(2) For any mini-versal openings $F:\left(\mathbf{R}^{n}, a\right) \rightarrow\left(\mathbf{R}^{m+r}, F(a)\right)$ and $F^{\prime}:\left(\mathbf{R}^{n}, a\right) \rightarrow\left(\mathbf{R}^{m+r}, F^{\prime}(a)\right)$ of $f$, there exists an affine bundle isomorphism $\Phi:\left(\mathbf{R}^{m+r}, F(a)\right) \rightarrow\left(\mathbf{R}^{m+r}, F^{\prime}(a)\right)$ over $\left(\mathbf{R}^{m}, f(a)\right)$ such that $F^{\prime}=\Psi \circ F$. In particular, the diffeomorphism class of mini-versal opening of $f$ is unique. (3) Any versal openings $F^{\prime \prime}:\left(\mathbf{R}^{n}, a\right) \rightarrow\left(\mathbf{R}^{m+s}, F^{\prime \prime}(a)\right)$ of $f$ is diffeomorphic to $(F, 0)$ for a miniversal opening of $f$.

Proof: (1) Let $F=\left(f, h_{1}, \ldots, h_{r}\right)$ and $G=\left(f, k_{1}, \ldots, k_{s}\right)$. Since $k_{j} \in \mathcal{R}_{f}$, there exist $c_{j}{ }^{0}, c_{j}{ }^{1}, \ldots, c_{j}{ }^{r} \in$ $\mathcal{E}_{b}$ such that $k_{j}=c_{j}{ }^{0} \circ f+\left(c_{j}{ }^{1} \circ f\right) h_{1}+\cdots+\left(c_{j}{ }^{r} \circ f\right) h_{r}$. Then it suffices to set $\Psi(y, z)=$ $\left(y,\left(c_{j}^{0}(y)+c_{j}^{1}(y) z_{1}+\cdots+c_{j}^{r}(y) z_{r}\right)_{1 \leq j \leq s}\right)$. (2) By (1) there exists an affine bundle map $\Psi$ with $F^{\prime}=\Psi \circ F$. From the minimality, we have that the matrix $\left(c_{j}^{i}(b)\right)$ is regular. (See Remark 6.5). Therefore $\Psi$ is a diffeomorphism-germ. (3) Let $F=\Psi \circ F^{\prime \prime}$ for some affine bundle map $\Psi$. Then the matrix $\left(c_{j}^{i}(b)\right)$ is of rank $r$. Therefore $F^{\prime \prime}$ is diffeomorphic to $\left(F, k_{1}, \ldots, k_{s-r}\right)$ for some $k_{j} \in \mathcal{R}_{f}$. Write each $k_{j}=K_{j} \circ F$ for some $K_{j} \in \mathcal{E}_{F(a)}$. Then we set $\Xi(y, z, w)=(y, z, w-K \circ F)$. Then $\Xi$ is a local diffeomorphism on $\mathbf{R}^{m+r+(s-r)}$ and $\Xi \circ\left(F, k_{1}, \ldots, k_{s-r}\right)=(F, 0)$.

\section{Normal forms of tangent surfaces.}

According to a geometric restriction expressed in differential system, we have imposed on curves in projective spaces a system of differential equations (\$3). The genericity, in such a restricted class of curves, naturally implies a restriction on types of curves (\$5). Then we use the following results to solve the classification problem. For the concrete expression of normal forms, see $\sqrt{3}$.

Theorem 7.1 (1) In $\mathbf{R} P^{3}$, the tangent variety of a curve of type $(1,2,3)$ (resp. $(1,2,4),(2,3,4)$, $(1,3,4))$ is locally diffeomorphic to the cuspidal edge (the folded umbrella, the swallowtail, the Mond surface) in $\mathbf{R}^{3}$.

(2) (Higher codimensional case.) In $\mathbf{R} P^{N+1}, N+1 \geq 4$,

(i) the tangent variety of a curve of type $\left(1,2,3, a_{4}, \ldots, a_{N+1}\right)$ is locally diffeomorphic to the cuspidal edge $\left(\mathbf{R}^{2}, 0\right) \rightarrow\left(\mathbf{R}^{3}, 0\right)$ composed with the inclusion to $\left(\mathbf{R}^{N+1}, 0\right)$.

(ii) the tangent variety of a curve of type $\left(1,3,4,5, a_{5}, \ldots, a_{N+1}\right)$ is locally diffeomorphic to the open Mond surface $\left(\mathbf{R}^{2}, 0\right) \rightarrow\left(\mathbf{R}^{4}, 0\right)$ composed with the inclusion to $\left(\mathbf{R}^{N+1}, 0\right)$.

(iii) the tangent variety of a curve of type $\left(2,3,4,5, a_{5}, \ldots, a_{N+1}\right)$ is locally diffeomorphic to the open swallowtail $\left(\mathbf{R}^{2}, 0\right) \rightarrow\left(\mathbf{R}^{4}, 0\right)$ composed with the inclusion to $\left(\mathbf{R}^{N+1}, 0\right)$. 
Proof: (1) is proved in Theorem $1(n=2)$ in [19]. (2) In each case, the idea is to show that the tangent map-germ $\operatorname{Tan}(\gamma)$ is diffeomorphic to a mini-versal opening of an appropriate map-germ:

(i) the fold map-germ $\left(\mathbf{R}^{2}, 0\right) \rightarrow\left(\mathbf{R}^{2}, 0\right)$.

(ii) the Mond surface $\left(\mathbf{R}^{2}, 0\right) \rightarrow\left(\mathbf{R}^{3}, 0\right)$.

(iii) the swallowtail $\left(\mathbf{R}^{2}, 0\right) \rightarrow\left(\mathbf{R}^{3}, 0\right)$.

Then, by Proposition 6.9, the diffeomorphism class of the tangent map-germ is unique and we get the required results.

Let $\gamma:(\mathbf{R}, 0) \rightarrow \mathbf{R} P^{N+1}$ be a curve-germ of type $\left(a_{1}, a_{2}, \ldots, a_{N+1}\right)$,

$$
\gamma(t)=\left(x_{1}(t), x_{2}(t), \ldots, x_{N+1}(t)\right)
$$

a local affine representation of $\gamma$ as in Lemma 4.2, and

$$
f(s, t)=\left(f_{1}(s, t), f_{2}(s, t), \ldots, f_{N+1}(s, t)\right)=\left(x_{i}(t)+s \frac{1}{\alpha(t)} x_{i}^{\prime}(t)\right)_{1 \leq i \leq N+1},
$$

the parametrisation of the tangent variety to $\gamma$, where $\alpha(t)=t^{a_{1}-1}$. We may suppose $x_{1}(t)=t^{a_{1}}$.

We define $g^{\prime}:\left(\mathbf{R}^{2}, 0\right) \rightarrow\left(\mathbf{R}^{2}, 0\right)$ by $g^{\prime}=\left(f_{1}, f_{2}\right)$. Then, by Lemma 4.5 and Remark 4.7, we see that $f_{3}, \ldots, f_{N+1} \in \mathcal{R}_{g^{\prime}}$. Note that $f_{1}(s, t)=x_{1}(t)+a_{1} s$ is a regular function. We regard $f_{1}(s, t)$ as an unfolding parameter $u$. Then there exist diffeomorphism-germ $\sigma:\left(\mathbf{R}^{2}, 0\right) \rightarrow\left(\mathbf{R}^{2}, 0\right)$ and $\tau:\left(\mathbf{R}^{2}, 0\right) \rightarrow\left(\mathbf{R}^{2}, 0\right)$ such that $\sigma$ is of form $\sigma(u, t)=\left(\sigma_{1}(u), t \sigma_{2}(u, t)\right)$ and $g=\tau \circ g^{\prime} \circ \sigma$ is equal to (i) $(u, t) \mapsto\left(u, t^{2}+u t\right)$, (ii) $(u, t) \mapsto\left(u, t^{3}+u t^{2}\right)$, (iii) $(u, t) \mapsto\left(u, t^{3}+u t\right)$. Then $f_{3} \circ \sigma, \ldots, f_{N+1} \circ \sigma$ belongs $\mathcal{R}_{g}=\mathcal{R}_{g^{\prime} \circ \sigma}$. Then, by Lemma 6.8. (i) $F=\left(f_{1} \circ \sigma, f_{2} \circ \sigma, f_{3} \circ \sigma\right)$, (ii)(iii) $F=\left(f_{1} \circ \sigma, f_{2} \circ \sigma, f_{3} \circ \sigma, f_{4} \circ \sigma\right)$, are versal opening of $g$ respectively. Note that in cases (ii) and (iii), $F$ is a versal opening of also Mond surface and swallowtail respectively. Then, by Proposition $6.8(3)$, we have that $f \circ \sigma$ is diffeomorphic to (i) $\left(u, t^{2}+u t, \frac{2}{3} t^{3}+\frac{1}{2} u t^{2}, 0, \ldots, 0\right)$, (ii) $\left(u, t^{3}+u t^{2}, \frac{3}{4} t^{4}+\frac{2}{3} u t^{3}, \frac{3}{5} t^{5}+\frac{1}{2} u t^{4}, 0, \ldots, 0\right)$, (iii) $\left(u, t^{3}+u t^{2}, \frac{3}{4} t^{4}+\frac{1}{2} u t^{2}, \frac{3}{5} t^{5}+\frac{1}{3} u t^{3}, 0, \ldots, 0\right)$, as required.

Theorem 7.2 The tangent variety of a curve of type $\left(1,2,4,5, a_{5}, \ldots, a_{N+1}\right)$ is locally diffeomorphic to the open folded umbrella $\left(\mathbf{R}^{2}, 0\right) \rightarrow\left(\mathbf{R}^{4}, 0\right)$ composed with the inclusion to $\left(\mathbf{R}^{N+1}, 0\right)$.

Proof: We argue as in Theorem 7.1. However in this case $\left(f_{1} \circ \sigma, f_{2} \circ \sigma, f_{3} \circ \sigma, f_{4} \circ \sigma\right)$ is not a versal opening of $g=\left(u, t^{2}+u t\right.$ ). (In fact the open folded umbrella is not a versal opening of the folded umbrella $\left(\mathbf{R}^{2}, 0\right) \rightarrow\left(\mathbf{R}^{3}, 0\right)$. $)$

To show Theorem 7.2 , we define

$$
\mathcal{R}_{g}^{(2)}:=\left\{h \in t^{2} \mathcal{E}_{2} \mid d h \in t^{2} \mathcal{J}_{g}\right\} .
$$

Then $f_{i} \circ \sigma \in \mathcal{R}_{g}^{(2)},(i \geq 3)$. We see that $f_{3} \circ \sigma, f_{4} \circ \sigma$ generate $\mathcal{R}_{g}^{(2)}$ over $g^{*} \mathcal{E}_{2}$. In fact $h_{1}, \ldots, h_{r}$ generate $\mathcal{R}_{g}^{(2)}$ as $\mathcal{E}_{2}$-module if and only if $i^{*} h_{1}, \ldots, i^{*} h_{r}$ generate $\mathfrak{m}_{1}^{4} / \mathfrak{m}_{1}^{6}$ over $\mathbf{R}$. (See Lemma 2.4 of [19]). Also $h_{1}=\frac{1}{2} t^{4}+\frac{1}{3} u t^{3}, h_{2}=\frac{2}{5} t^{5}+\frac{1}{4} u t^{4}$ generate $\mathcal{R}_{g}^{(2)}$. We write $f_{i} \circ \sigma=\left(a_{i} \circ g\right) h_{1}+\left(b_{i} \circ g\right) h_{2},(i \geq$ $3)$, for some $a_{i}, b_{i} \in \mathcal{E}_{2}$. We define $\Psi:\left(\mathbf{R}^{N+1}, 0\right) \rightarrow\left(\mathbf{R}^{N+1}, 0\right)$ by

$$
\begin{gathered}
\Psi(x)=\left(x_{1}, x_{2}, a_{3}\left(x_{1}, x_{2}\right) x_{3}+b_{3}\left(x_{1}, x_{2}\right) x_{4}, a_{4}\left(x_{1}, x_{2}\right) x_{3}+b_{4}\left(x_{1}, x_{2}\right) x_{4},\right. \\
\left.x_{i}-a_{i}\left(x_{1}, x_{2}\right) x_{3}+b_{i}\left(x_{1}, x_{2}\right) x_{4}(5 \geq i)\right) .
\end{gathered}
$$

Then $\Psi$ is a diffeomorphism-germ and $\Psi \circ f \circ \sigma=\left(g, h_{1}, h_{2}, 0\right)$. Thus we have that $f \circ \sigma$ is diffeomorphic to $\left(g, h_{1}, h_{2}, 0\right)=\left(u, t^{2}+u t, \frac{1}{2} t^{4}+\frac{1}{3} u t^{3}, \frac{2}{5} t^{5}+\frac{1}{4} u t^{4}, 0, \ldots, 0\right)$ as required.

Proofs of the classification theorems. Theorems 2.6, 3.3, 3.4, 3.6 follows from Theorems 5.8, 5.9, 5.5 and Theorems 7.1, 7.2. 
We are led, in our generic classifications in a geometric setting, to find the following result, which we use in $\$ 8$.

Theorem 7.3 The tangent variety of a curve of type $\left(1,3,4,6, a_{5}, \ldots, a_{N+1}\right)$ in $\mathbf{R} P^{N+1}, N+1 \geq 4$, has unique diffeomorphism class.

We may call it the 'unfurled Mond surface', distinguished with the open Mond surface. The normal form $\left(\mathbf{R}^{2}, 0\right) \rightarrow\left(\mathbf{R}^{N+1}, 0\right)$ of the unfurled Mond surface is given by

$$
(s, t) \mapsto\left(t+s, t^{3}+3 s t^{2}, t^{4}+4 s t^{3}, t^{6}+6 s t^{5}, 0, \ldots, 0\right),
$$

which is diffeomorphic to

$$
(x, u) \mapsto\left(u, t^{3}+u t^{2}, \frac{3}{4} t^{4}+\frac{2}{3} u t^{3}, \frac{1}{2} t^{6}+\frac{2}{5} u t^{5}, 0, \ldots, 0\right) .
$$

To show Theorem 7.3 , we prepare the following:

Lemma 7.4 (cf. Lemma 2.4 of [19]) Let $g:\left(\mathbf{R}^{2}, 0\right) \rightarrow\left(\mathbf{R}^{2}, 0\right)$ be the map-germ defined by $g(t, u)=$ $\left(u, t^{3}+u t^{2}\right)$. We set

$$
\mathcal{R}_{g}^{(3)}:=\left\{h \in t^{3} \mathcal{E}_{2} \mid d h \in t^{3} \mathcal{J}_{g}\right\} .
$$

and set $T=t^{3}+u t^{2}, T_{i}=\frac{3}{i+3} t^{i+3}+\frac{2}{i+2} u t^{i+2},(i=1,2,3, \ldots)$. Then we have (1) $\mathcal{R}_{g}^{(3)}=\mathcal{R}_{g} \cap t^{5} \mathcal{E}_{2}$. (2) $\mathcal{R}_{g}^{(3)}$ is a finite $\mathcal{E}_{2}$-module via $g^{*}: \mathcal{E}_{2} \rightarrow \mathcal{E}_{2}$ generated by $T_{3}, T T_{1}, T_{1}^{2}$. (3) Let $\iota:(\mathbf{R}, 0) \rightarrow$ $\left(\mathbf{R}^{2}, 0\right), \iota(t)=(t, 0)$. Then $h_{1}, \ldots, h_{\ell} \in \mathcal{R}_{g}^{(3)}$ generate $\mathcal{R}_{g}^{(3)}$ as $\mathcal{E}_{2}$-module via $g^{*}$ if and only if $\iota^{*} h_{1}, \ldots, \iota^{*} h_{\ell}$ generate $t^{6} \mathcal{E}_{1} / t^{9} \mathcal{E}_{1}$ over $\mathbf{R}$. (Note that $T_{1} \notin \mathcal{R}_{g}^{(3)}$.)

Proof: (1) First note that $\mathcal{R}_{g}^{(3)}=\left\{h \in t^{3} \mathcal{E}_{2} \mid \frac{\partial h}{\partial t} \in t^{3} \frac{\partial T}{\partial t} \mathcal{E}_{2}\right\}$. Let $h \in \mathcal{R}_{g}^{(3)}$. Then $\frac{\partial h}{\partial t} \in t^{4} \mathcal{E}_{2}$ and $h \in t^{3} \mathcal{E}_{2}$. Therefore $h \in \mathcal{R}_{g} \cap t^{5} \mathcal{E}_{2}$. Conversely let $h \in \mathcal{R}_{g} \cap t^{5} \mathcal{E}_{2}$. Then $\frac{\partial h}{\partial t}=t^{3} \frac{\partial T}{\partial t} K$ for some $K \in \mathcal{E}_{2}$. Since $h(0,0)=0$, we have $\frac{\partial h}{\partial u} \in t^{5} \mathcal{E}_{2}$. Therefore $d h \in t^{3} \mathcal{J}_{g}$ and $h \in \mathcal{R}_{g}^{(3)}$. Thus we have the equality.

(2) Let $h \in \mathcal{R}_{g}^{(3)}$. Then $h=a \circ g+b \circ g T_{1}+c \circ g T_{2}$, for some $a, b, c \in \mathcal{E}_{2}$. Since $h \in t^{5} \mathcal{E}_{2}$, $h=\widetilde{a} \circ g T^{3}+\widetilde{b} \circ g T T_{1}+\widetilde{c} \circ g T T_{2}$, for some $\widetilde{a}, \widetilde{b}, \widetilde{c} \in \mathcal{E}_{2}$. Note that $T^{3}, T T_{1}, T T_{2} \in \mathcal{R}_{g}^{(3)}$. Moreover we have directly

$$
T^{3}=\frac{32}{15} u T_{1}^{2}+2 T T_{3}+\frac{14}{3} T_{4}, T T_{2}=\frac{16}{15} T_{1}^{2}+\frac{7}{3} u T_{4}, T_{4}=\frac{4}{7} T T_{1}-\frac{20}{21} u T_{3} .
$$

Therefore we have

$$
T T_{2}=-\frac{20}{9} u^{2} T_{3}+\frac{4}{3} u T T_{1}+\frac{16}{15} T_{1}^{2}, T^{3}=\left(2 T-\frac{40}{9} u^{3}\right) T_{3}+\frac{8}{3} u^{2} T T_{1}+\frac{32}{15} u T_{1}^{2} .
$$

(3) $\iota^{*}: \mathcal{E}_{2} \rightarrow \mathcal{E}_{1}$ induces $\iota^{*}: \mathcal{R}_{g}^{(3)} \rightarrow t^{6} \mathcal{E}_{1}$, which is clearly surjective. Moreover we have $\left(\iota^{*}\right)^{-1}\left(t^{9} \mathcal{E}_{1}\right)=g^{*} \mathfrak{m}_{2} \mathcal{R}_{g}^{(3)}$. Therefore $\iota^{*}$ induces an isomorphism $\mathcal{R}_{g}^{(3)} / g^{*} \mathfrak{m}_{2} \mathcal{R}_{g}^{(3)} \cong t^{6} \mathcal{E}_{1} / t^{9} \mathcal{E}_{1}$ as $\mathbf{R}$ vector spaces. By (2) and by Malgrange-Mather's preparation theorem [5], we have the required result.

Proof of Theorem 7.3: We give the proof for the case $N+1=4$. In general case we can argue similarly. 
Let $\gamma:(\mathbf{R}, 0) \rightarrow \mathbf{R} P^{4}$ be a curve of type $(1,3,4,6)$. The tangent map-germ $\operatorname{Tan}(\gamma)$ is an opening of a Mond surface. However it is not versal. So we need a specialised idea to show the determinacy result in this situation. Let

$$
\gamma(t)=\left(t, t^{3}+\varphi(t), t^{4}+\psi(t), t^{6}+\rho(t)\right),
$$

with $\varphi \in \mathfrak{m}_{1}^{4}, \psi \in \mathfrak{m}_{1}^{5}, \rho \in \mathfrak{m}_{1}^{7}$. Then $f=\operatorname{Tan}(\gamma)$ is given by

$$
f(s, t)=\left(t+s, t^{3}+3 s t^{2}+\Phi(t), t^{4}+4 s t^{3}+\Psi(t), t^{6}+6 s t^{5}+R(t)\right),
$$

where $\Phi(s, t)=\varphi(t)+s \varphi^{\prime}(t), \Psi(s, t)=\psi(t)+s \psi^{\prime}(t), R(s, t)=\rho(t)+s \rho^{\prime}(t)$. We set $u=t+s$. Then

$$
f(u, t)=\left(u,-2 t^{3}+3 u t^{2}+\widetilde{\Phi}(t),-3 t^{4}+4 u t^{3}+\widetilde{\Psi}(t),-5 t^{6}+6 u t^{5}+\widetilde{R}(t)\right),
$$

where $\Phi(s, t)=\varphi(t)+(u-t) \varphi^{\prime}(t), \Psi(s, t)=\psi(t)+(u-t) \psi^{\prime}(t), R(s, t)=\rho(t)+(u-t) \rho^{\prime}(t)$. From the determinacy of tangent varieties to curves of type $(1,3,4)$ in $\mathbf{R}^{3}$ ([33], [17]), we have that there exist diffeomorphism-germ $\sigma:\left(\mathbf{R}^{2}, 0\right) \rightarrow\left(\mathbf{R}^{2}, 0\right)$ of form $\sigma(u, t)=\left(\sigma_{1}(u), t \sigma_{2}(u, t)\right)$ and a diffeomorphism-germ $\tau:\left(\mathbf{R}^{4}, 0\right) \rightarrow\left(\mathbf{R}^{4}, 0\right)$ such that

$$
f \circ \sigma(u, t)=\left(u, T(u, t), T_{1}(u, t), T_{3}(u, t)+S_{3}(u, t)\right)
$$

with

$$
T=t^{3}+u t^{2}, \quad T_{1}=\frac{3}{4} t^{4}+\frac{2}{3} u t^{3}, \quad T_{3}=\frac{1}{2} t^{6}+\frac{2}{5} u t^{5},
$$

$S_{3} \in \mathcal{R}_{g}^{(3)}, g=\left(u, t^{3}+u t^{2}\right), \iota^{*} S_{3} \in \mathfrak{m}_{1}^{7}$. Then we have, by Lemma 7.4 .

$$
S_{3}=A_{3} \circ g T_{3}+B_{3} \circ g T T_{1}+C_{3} \circ g T_{1}^{2},
$$

for some $A_{3}, B_{3}, C_{3} \in \mathcal{E}_{2}$ with $A_{3}(0,0)=0$. Define $\Xi:\left(\mathbf{R}^{4}, 0\right) \rightarrow\left(\mathbf{R}^{4}, 0\right)$ by

$$
\begin{array}{r}
\Xi\left(x_{1}, x_{2}, x_{3}, x_{4}\right)=\left(x_{1}, x_{2}, x_{3}+A_{1}\left(x_{1}, x_{2}\right) x_{4}+B_{1}\left(x_{1}, x_{2}\right) x_{2} x_{3}+C_{1}\left(x_{1}, x_{2}\right) x_{3}^{2},\right. \\
\left.x_{4}+A_{3}\left(x_{1}, x_{2}\right) x_{4}+B_{3}\left(x_{1}, x_{2}\right) x_{2} x_{3}+C_{3}\left(x_{1}, x_{2}\right) x_{3}^{2}\right) .
\end{array}
$$

Then the Jacobi matrix of $\Xi$ is the unit matrix, so $\Xi$ is a diffeomorphism-germ and

$$
\Xi^{-1} \circ f \circ \sigma=\left(u, t^{3}+u t^{2}, \frac{3}{4} t^{4}+\frac{2}{3} u t^{3}, \frac{1}{2} t^{6}+\frac{2}{5} u t^{5}\right) .
$$

\section{Singularities on tangent varieties to osculating-framed contact- integral curves.}

We give results on the classification of singularities of tangent varieties to contact-integral curves (resp. osculating framed contact-integral curves) in a contact projective space.

Let $V$ be a symplectic vector space of dimension $2 n+2$. Consider the isotropic flag manifold:

$$
\mathcal{F}_{\text {Lag }}=\mathcal{F}_{\text {Lag }}(V):=\left\{V_{1} \subset V_{2} \subset \cdots \subset V_{n} \subset V_{n+1} \subset V \mid V_{n+1} \text { is Lagrangian }\right\} .
$$

Note that $\mathcal{F}_{\text {Lag }}$ is a finite quotient of $\mathrm{U}(n+1), \operatorname{dim}\left(\mathcal{F}_{\text {Lag }}\right)=(n+1)^{2}$ and that $\mathcal{F}_{\text {Lag }}(V)$ is embedded into $\mathcal{F}(V)=\mathcal{F}_{1,2, \ldots, n+1, \ldots, 2 n+1}(V)$ by taking symplectic orthogonals:

$$
\left(V_{1}, V_{2}, \ldots, V_{n}, V_{n+1}\right) \mapsto\left(V_{1}, V_{2}, \ldots, V_{n}, V_{n+1}, V_{n}^{s}, \ldots, V_{2}^{s}, V_{1}^{s}\right),
$$


Define a differential system $\mathcal{E} \subset T \mathcal{F}_{\text {Lag }}$ by

$$
v \in \mathcal{E}_{\left(V_{1}, \ldots, V_{n+1}\right)} \Longleftrightarrow \pi_{i *}(v) \in T \operatorname{Gr}\left(i, V_{i+1}\right)(\subset T \operatorname{IGr}(i, V)),(1 \leq i \leq n) .
$$

where IGr means the isotropic Grassmannian, $\pi_{i}: \mathcal{F}_{\text {Lag }} \rightarrow \operatorname{IGr}(i, V)$ is the canonical projection. Then $\operatorname{rank}(\mathcal{E})=n+1$ and $\mathcal{E}$ is bracket generating.

If $n=1$, then we have $\operatorname{dim} \mathcal{F}_{\text {Lag }}=4$ and $\mathcal{E}$ is an Engel structure on $\mathcal{F}_{\text {Lag }}([15])$.

An $\mathcal{E}$-integral curve $c: I \rightarrow \mathcal{F}_{\text {Lag }}$ is a $C^{\infty}$ family

$$
\left(V_{1}(t), V_{2}(t), \ldots, V_{n}(t), V_{n+1}(t)\right)
$$

of isotropic flags in the symplectic vector space $V$ such that $V_{i}(t)$ moves momentarily in $V_{i+1}(t)$.

Remark 8.1 The projective space $P\left(V^{2 n+2}\right) \cong \mathbf{R} P^{2 n+1}$ has the canonical contact structure $\mathcal{D} \subset$ $T(P(V))$ : For $V_{1} \in P(V)$ and for $v \in T_{V_{1}} P(V)$, we define

$$
v \in \mathcal{D}_{V_{1}} \Longleftrightarrow \pi_{1 *}(v) \in T\left(P\left(V_{1}^{s}\right)\right)(\subset T(P(V))) .
$$

If $c: I \rightarrow \mathcal{F}_{\mathrm{Lag}}(V)$ is an $\mathcal{E}$-integral curve, then $\gamma=\pi_{1} \circ c: I \rightarrow P(V)$ is a $\mathcal{D}$-integral curve.

We consider the space $J_{\mathcal{E}}^{r}\left(I, \mathcal{F}_{\text {Lag }}\left(\mathbf{R}^{2 n+2}\right)\right.$ of $\mathcal{E}$-integral jets in $J^{r}\left(I, \mathcal{F}_{\mathrm{Lag}}\left(\mathbf{R}^{2 n+2}\right)\right)$ and set

$$
\Sigma_{\mathcal{E}}(\mathbf{A}):=\left\{j^{r} \Gamma\left(t_{0}\right) \mid t_{0} \in I, \Gamma:\left(\mathbf{R}, t_{0}\right) \rightarrow \mathcal{F}_{\text {Lag }}\left(\mathbf{R}^{2 n+2}\right) \text { is } \mathcal{E} \text {-integral, } \pi_{1} \circ \Gamma \text { is of type } \mathbf{A}\right\} .
$$

Then we have the codimension formula for osculating framed contact-integral curves.

Theorem 8.2 The set of $\mathcal{E}$-integral curves $c: I \rightarrow \mathcal{F}_{\text {Lag }}\left(\mathbf{R}^{2 n+2}\right)$ such that the osculating-framed contact-integral curve $\pi_{1} \circ c: I \rightarrow P\left(V^{2 n+2}\right)$ is of type $\mathbf{A}=\left(a_{1}, a_{2}, \ldots, a_{2 n+1}\right)$ is not empty if and only if

$$
a_{n+j}=a_{n+1}+a_{n}-a_{n+1-j}, \quad(2 \leq j \leq n+1),
$$

and then its codimension in the jet space of $\mathcal{E}$-integral curves is given by $a_{n+1}-(n+1)$.

Proof: To show Theorem 8.2, first we give systems of projective coordinates on $\mathcal{F}_{\text {Lag }}(V)$. For the case $n=1$, refer the paper [15].

We fix a flag $\mathbf{V}_{0}=\left(V_{10}, V_{20}, \ldots, V_{n+10}\right) \in \mathcal{F}_{\text {Lag }}(V)$. Then we take the open set $U \subset \mathcal{F}_{\text {Lag }}(V)$ defined by

$$
U:=\left\{\left(V_{1}, V_{2}, \ldots, V_{n+1}\right) \in \mathcal{F}_{\text {Lag }}(V) \mid V_{1} \cap V_{10}^{s}=\{0\}, V_{2} \cap V_{20}^{s}=\{0\}, \ldots, V_{n+1} \cap V_{n+10}^{s}=\{0\}\right\} .
$$

Take $\mathbf{V}_{1}=\left(V_{11}, V_{21}, \ldots, V_{n+11}\right) \in U$. Then we have the decomposition $V=V_{n+11} \oplus V_{n+10}$ into Lagrangian subspaces, and the decomposition

$$
\begin{aligned}
& V_{n+11}=V_{11} \oplus\left(V_{21} \cap V_{10}^{s}\right) \oplus\left(V_{31} \cap V_{20}^{s}\right) \oplus \cdots \oplus\left(V_{n+11} \cap V_{n 0}^{s}\right), \\
& V_{n+10}=V_{10} \oplus\left(V_{20} \cap V_{11}^{s}\right) \oplus\left(V_{30} \cap V_{21}^{s}\right) \oplus \cdots \oplus\left(V_{n+10} \cap V_{n 1}^{s}\right),
\end{aligned}
$$

of each Lagrangian subspace into one-dimensional subspaces. Take non-zero vectors $e_{0} \in V_{11}$, $e_{i} \in V_{i+11} \cap V_{i 0}^{s},(1 \leq i \leq n), f_{0} \in V_{10}$ and $f_{i} \in V_{i+10} \cap V_{i 1}^{s},(1 \leq i \leq n)$, to get a symplectic basis $\left(e_{0}, e_{1}, \ldots, e_{n} ; f_{0}, f_{1}, \ldots, f_{n}\right)$ of $V$.

Then, for each $\mathbf{V}=\left(V_{1}, V_{2}, \ldots, V_{n+1}\right) \in U_{\mathbf{V}_{0}}, V_{n+1}$ has a basis $\left(v_{0}, v_{1}, \ldots, v_{n}\right)$ uniquely expressed as

$$
v_{i}=e_{i}+\sum_{j=0}^{n} x_{j}{ }^{i} f_{j}, \quad(0 \leq i \leq n),
$$


for some $\left(x_{j}{ }^{i}\right)_{0 \leq i, j \leq n}$. Since $V_{n+1}$ is a Lagrangian subspace of $V$, we have that $x_{j}{ }^{i}=x_{i}{ }^{j}, 0 \leq i, j \leq n$. Then there exist uniquely $\lambda_{i}{ }^{k},(1 \leq k \leq i \leq n)$, such that

$$
w_{k}=v_{k-1}+\sum_{i=k}^{n} \lambda_{i}{ }^{k} v_{i}, \quad(1 \leq k \leq n+1),
$$

form a basis of $V_{n+1}$ such that $V_{k}=\left\langle w_{1}, \ldots, w_{k}\right\rangle_{\mathbf{R}},(1 \leq k \leq n+1)$. Then actually we have

$$
w_{k}=e_{k-1}+\sum_{i=k}^{n} \lambda_{i}{ }^{k} e_{i}+\sum_{j=0}^{n}\left(x_{j}^{k-1}+\sum_{i=k}^{n} \lambda_{i}{ }^{k} x_{j}^{i}\right) f_{j}, \quad(1 \leq k \leq n+1) .
$$

Thus, given $\mathbf{V}_{0}, \mathbf{V}_{1} \in \mathcal{F}_{\text {Lag }}(V)$, we have a chart $U \rightarrow \mathbf{R}^{(n+1)^{2}}$ of $\mathcal{F}_{\mathrm{Lag}}(V)$, given by the symmetric matrix $\left(x_{j}{ }^{i}\right)_{0 \leq i, j \leq n}$ and $\lambda_{i}{ }^{k},(1 \leq k \leq i \leq n)$. From another choice of $\mathbf{V}_{0}, \mathbf{V}_{1} \in \mathcal{F}_{\text {Lag }}(V)$, we have another chart with fractional linear transition functions.

The projection $\pi_{1}: \mathcal{F}_{\mathrm{Lag}}(V) \rightarrow P(V)$ is expressed by

$$
\left(x_{j}{ }^{i}, \lambda_{i}{ }^{k}\right) \mapsto\left[1: \lambda_{1}{ }^{1}: \cdots: \lambda_{1}{ }^{n}: x_{0}{ }^{0}+\sum_{i=1}^{n} \lambda_{i}{ }^{1} x_{0}{ }^{i}: \cdots: x_{n}{ }^{0}+\sum_{i=1}^{n} \lambda_{i}{ }^{1} x_{n}{ }^{i}\right] .
$$

We set $X_{j}{ }^{k}:=x_{j}{ }^{k}+\sum_{i=k+1}^{n} \lambda_{i}{ }^{k+1} x_{j}{ }^{i},(0 \leq j \leq n, 0 \leq k \leq n)$. Then the differential system $\mathcal{E}$ is locally given by

$$
\left\{\begin{array}{l}
d \lambda_{i}{ }^{k}-\lambda_{i}{ }^{k+1} d \lambda_{k}^{k}=0, \quad 1 \leq k \leq n, k+1 \leq i \leq n, \\
d X_{j}{ }^{k-1}-X_{j}{ }^{k} d \lambda_{k}^{k}=0, \quad 1 \leq k \leq n, 0 \leq j \leq n .
\end{array}\right.
$$

We see that each $\mathcal{E}$-integral curve $\Gamma$ is obtained from the components $\lambda_{k}{ }^{k}, 1 \leq k \leq n$, and the $x_{n}{ }^{n}$-component, by iterative integrations.

The type $\left(a_{1}, a_{2}, \ldots, a_{n}, a_{n+1}, a_{n+2}, \ldots, a_{2 n+1}\right)$ of $\gamma=\pi_{1} \circ \Gamma$ is expressed in terms of

$$
u_{k}:=\operatorname{ord}\left(\lambda_{k}^{k}\right), 1 \leq k \leq n, \quad v:=\operatorname{ord}\left(x_{n}{ }^{n}\right)
$$

by

$$
\begin{aligned}
a_{i} & =u_{1}+u_{2}+\cdots+u_{i},(1 \leq i \leq n) \\
a_{n+1} & =u_{1}+u_{2}+\cdots+u_{n}+v \\
a_{n+1+j} & =u_{1}+u_{2}+\cdots+2 u_{n-j+1}+\cdots+2 u_{n}+v,(1 \leq j \leq n),
\end{aligned}
$$

Let $\mathbf{A}=\left(a_{1}, \ldots, a_{n}, a_{n+1}, a_{n+2}, \ldots, a_{2 n}, a_{2 n+1}\right)$ be a strictly increasing sequence of positive integers. Then The above system of equations has an integer solution $\left(u_{1}, \ldots, u_{n}, v\right)$ if and only if $a_{n+1+i}-$ $a_{n+i}=a_{n}-a_{n-i}$. If the non-empty condition is fulfilled, then the codimension of the set

$$
\Sigma(\mathbf{A})=\left\{j^{r} \Gamma\left(t_{0}\right) \mid \Gamma:\left(I, t_{0}\right) \rightarrow \mathcal{F}_{\mathrm{Lag}}(V) \text { is } \mathcal{E} \text {-integral, type }\left(\pi_{1} \circ \Gamma\right)=\mathbf{A}\right\}
$$

in $J_{\mathcal{E}}^{r}\left(I, \mathcal{F}_{\mathrm{Lag}}(V)\right)$ is calculated by

$$
a_{1}-1+\left(a_{2}-a_{1}-1\right)+\cdots+\left(a_{n+1}-a_{n}-1\right)=a_{n+1}-(n+1) .
$$

By Theorem 8.2 and by the transversality theorem for $\mathcal{E}$-integral curves, we have the following result: We separate cases into three groups from the classification viewpoint of singularities. 

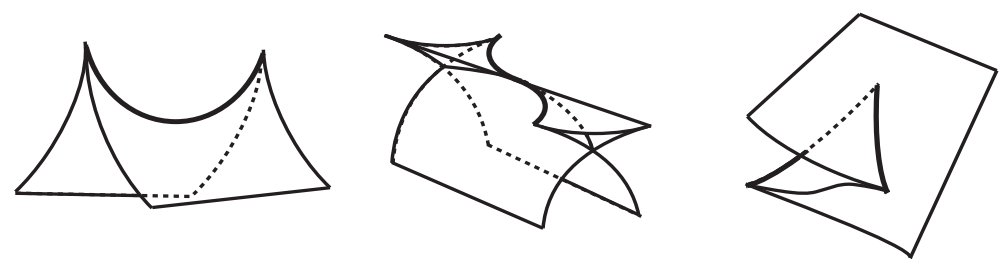

Figure 5: cuspidal edge, Mond surface and generic folded pleat in $\mathbf{R}^{3}$.

Theorem 8.3 ([15]) Let $2 n+1=3$. For a generic $\mathcal{E}$-integral curve $c: I \rightarrow \mathcal{F}_{\text {Lag }}\left(\mathbf{R}^{4}\right)$ in $C^{\infty}$ topology, the type $\mathbf{A}$ of $\pi_{1} \circ c$ at any point $t \in I$ is given by

$$
\mathbf{A}=(1,2,3),(1,3,4),(2,3,5) .
$$

The tangent varieties to the osculating-framed Legendre curve $\gamma=\pi_{1} \circ c: I \rightarrow P(V) \cong \mathbf{R} P^{3}$ is locally diffeomorphic to the cuspidal edge, to the Mond surface or to the generic folded pleat (Figure 5).

Remark 8.4 In the above Theorem 8.3, the type of the curve $\gamma$ is restricted to $(1,2,3),(1,3,4)$ or $(2,3,5)$. The local diffeomorphism class of the tangent variety $\operatorname{Tan}(\gamma)$ is determined if $\operatorname{type}(\gamma)=$ $(1,2,3)$ or $(1,3,4)$, but it is not determined if type $(\gamma)=(2,3,5)$ and there are exactly two diffeomorphism classes, generic one and non-generic one.

Note that we have obtained in [15] also the generic classification of singularities of tangent varieties to $\pi_{2} \circ c: I \rightarrow \mathrm{LG}(V)$ in Lagrangian Grassmannian.

In the higher codimensional case, we have:

Theorem 8.5 Let $2 n+1 \geq 7$. For a generic $\mathcal{E}$-integral curve $c: I \rightarrow \mathcal{F}_{\mathrm{Lag}}\left(\mathbf{R}^{2 n+2}\right)$ in $C^{\infty}$-topology, the type of osculating-framed contact-integral curve $\gamma=\pi_{1} \circ c: I \rightarrow P(V) \cong \mathbf{R} P^{2 n+1}$ at each point of $I$ is given by one of

$$
\begin{aligned}
\mathbf{A}=\quad & (1,2,3,4, \ldots, n, n+1, n+2, \ldots, 2 n+1), \\
& (1,2,3,4, \ldots, n, \quad n+2, n+3, \ldots, 2 n+2), \\
& \ldots \ldots \ldots \\
& (1,2,4,5, \ldots, n+1, n+2, n+3, \ldots, 2 n+2), \\
& (1,3,4,5, \ldots, n+1, n+2, n+3, \ldots, 2 n+2), \\
& (2,3,4,5, \ldots, n+1, n+2, n+3, \ldots, 2 n+2) .
\end{aligned}
$$

Moreover the tangent variety $\operatorname{Tan}(\gamma)$ to the osculating-framed contact-integral curve $\gamma$ is locally diffeomorphic to the cuspidal edge, the open folded umbrella, the open Mond surface, or to the open swallowtail.

We should be careful in the low codimensional case:

Theorem 8.6 Let $2 n+1=5$. For a generic $\mathcal{E}$-integral curve $c: I \rightarrow \mathcal{F}_{\text {Lag }}\left(\mathbf{R}^{6}\right)$ in $C^{\infty}$-topology, the type of osculating-framed contact-integral curve $\gamma=\pi_{1} \circ c: I \rightarrow P(V) \cong \mathbf{R} P^{5}$ at each point of $I$ is given by one of

$$
(1,2,3,4,5),(1,2,4,5,6),(1,3,4,6,7),(2,3,4,5,7) .
$$

Moreover the tangent variety $\operatorname{Tan}(\gamma)$ to the osculating-framed contact-integral curve $\gamma$ is locally diffeomorphic to the cuspidal edge, the open folded umbrella, the unfurled Mond surface, or to the open swallowtail. 
Proofs of Theorems 8.5, 8.6: By the transversality theorem, we reduce the list in each case from Theorem 8.2. In each case, we have the uniqueness of the diffeomorphism class of tangent varieties by Theorem 7.1, except for the case $\mathbf{A}=(1,3,4,6,7)$. For the case $\mathbf{A}=(1,3,4,6,7)$, we use Theorem 7.3 .

It is natural to consider the generic classification of tangent varieties to contact-integral curves $I \rightarrow P(V)=\mathbf{R} P^{2 n+1}$. Here, we give just the result on non-framed three dimensional case $(n=1)$ :

Proposition 8.7 For a generic contact-integral curve $\gamma: I \rightarrow P\left(V^{4}\right) \cong \mathbf{R} P^{3}$, and for any $t_{0} \in I$, the type of $\gamma$ at $t_{0}$ is equal to $(1,2,3)$ or to $(1,3,4)$ and the tangent variety $\operatorname{Tan}(\gamma)$ of $\gamma$ is locally diffeomorphic to the cuspidal edge or to the Mond surface.

Proof: Take the local coordinates $\lambda, \mu, \nu$ of $P(V)$ such that the contact structure is given by $d \mu=$ $\nu d \lambda-\lambda d \nu$. We express $\gamma(t)=(\lambda(t), \mu(t), \nu(t))$. Since $\gamma$ is contact-integral, we have that $\mu^{\prime}(t)=$ $\nu(t) \lambda^{\prime}(t)-\lambda(t) \nu^{\prime}(t)$. Therefore $\mu^{\prime \prime}(t)=\nu(t) \lambda^{\prime \prime}(t)-\lambda(t) \nu^{\prime \prime}(t)$ and

$$
\mu^{\prime \prime \prime}(t)=\nu^{\prime}(t) \lambda^{\prime \prime}(t)+\nu(t) \lambda^{\prime \prime \prime}(t)-\lambda^{\prime}(t) \nu^{\prime \prime}(t)-\lambda(t) \nu^{\prime \prime \prime}(t) .
$$

Then

$$
\operatorname{det}\left(\begin{array}{ccc}
\lambda^{\prime} & \mu^{\prime} & \nu^{\prime} \\
\lambda^{\prime \prime} & \mu^{\prime \prime} & \nu^{\prime \prime} \\
\lambda^{\prime \prime \prime} & \mu^{\prime \prime \prime} & \nu^{\prime \prime \prime}
\end{array}\right)=\left(\lambda^{\prime} \nu^{\prime \prime}-\lambda^{\prime \prime} \nu^{\prime}\right)^{2}
$$

Therefore, if type $(\lambda(t), \nu(t))=(1,2)$, then type $(\gamma(t))=(1,2,3)$. Moreover we have

$$
\mu^{\prime \prime \prime \prime}=2 \nu^{\prime} \lambda^{\prime \prime \prime}+\nu \lambda^{\prime \prime \prime \prime}-2 \lambda^{\prime} \nu^{\prime \prime \prime}-\lambda \nu^{\prime \prime \prime \prime} \text {. }
$$

Then

$$
\operatorname{rank}\left(\begin{array}{ccc}
\lambda^{\prime} & \mu^{\prime} & \nu^{\prime} \\
\lambda^{\prime \prime} & \mu^{\prime \prime} & \nu^{\prime \prime} \\
\lambda^{\prime \prime \prime} & \mu^{\prime \prime \prime} & \nu^{\prime \prime \prime} \\
\lambda^{\prime \prime \prime \prime} & \mu^{\prime \prime \prime \prime} & \nu^{\prime \prime \prime \prime}
\end{array}\right)=\operatorname{rank}\left(\begin{array}{ccc}
\lambda^{\prime} & \nu^{\prime} & 0 \\
\lambda^{\prime \prime} & \nu^{\prime \prime} & 0 \\
\lambda^{\prime \prime \prime} & \nu^{\prime \prime \prime} & \lambda^{\prime} \nu^{\prime \prime}-\lambda^{\prime \prime} \nu^{\prime} \\
\lambda^{\prime \prime \prime \prime} & \nu^{\prime \prime \prime \prime} & \lambda^{\prime} \nu^{\prime \prime \prime}-\lambda^{\prime \prime \prime} \nu^{\prime}
\end{array}\right) .
$$

Therefore the rank of the above matrix is 3 at $t$ if and only if $\lambda^{\prime} \nu^{\prime \prime}-\lambda^{\prime \prime} \nu^{\prime} \neq 0$ or $\lambda^{\prime} \nu^{\prime \prime \prime}-\lambda^{\prime \prime \prime} \nu^{\prime} \neq 0$ at $t$. By the transversality theorem, we have that, for a generic $\gamma$ and for any $t_{0} \in I$, (a) $\lambda^{\prime}\left(t_{0}\right) \nu^{\prime \prime}\left(t_{0}\right)-$ $\lambda^{\prime \prime}\left(t_{0}\right) \nu^{\prime}\left(t_{0}\right) \neq 0$ or (b) $\lambda^{\prime}\left(t_{0}\right) \nu^{\prime \prime}\left(t_{0}\right)-\lambda^{\prime \prime}\left(t_{0}\right) \nu^{\prime}\left(t_{0}\right)=0$ and $\lambda^{\prime}\left(t_{0}\right) \nu^{\prime \prime \prime}\left(t_{0}\right)-\lambda^{\prime \prime \prime}\left(t_{0}\right) \nu^{\prime}\left(t_{0}\right) \neq 0$. In case $(\mathrm{a}), \operatorname{type}(\gamma)=(1,2,3)$ at $t_{0}$. In case $(\mathrm{b}), \operatorname{type}(\gamma)=(1,3,4)$ at $t_{0}$. Then, by Theorem $7.1(1)$, we have the required result.

\section{Singularities of tangent varieties to surfaces.}

First we observe that the tangent varieties to a generic smooth surface are not frontal.

Example 9.1 Let $V=\left\{A=\left(\begin{array}{ccc}a_{11} & a_{12} & a_{13} \\ a_{12} & a_{22} & a_{23} \\ a_{13} & a_{23} & a_{33}\end{array}\right) \mid 3 \times 3\right.$, symmetric $\}$,

the vector space of quadratic form of variables $x, y, z$. Then $\operatorname{dim}(V)=6$. Let $S=P(\{\operatorname{rank}(A)=$ $1\}) \subset P(V) \cong \mathbf{R} P^{5}$ be the Veronese surface. Then we see that the tangent variety consists of the projection of the locus of semi-indefinite matrices of rank 2 and $S$. Note that the secant variety $\operatorname{Sec}(S)$, the closure of the union of secants connecting any pair of points on $S$, consists of the projection of the locus of matrices of rank $\leq 2$ :

$$
\begin{gathered}
\operatorname{Tan}(S)=S \cup P(\{\operatorname{rank}(A)=2, \text { semi-indefinite }\}) \\
\subsetneq \operatorname{Sec}(S)=P(\{\operatorname{rank}(A) \leq 2\}) \subsetneq P(V) .
\end{gathered}
$$




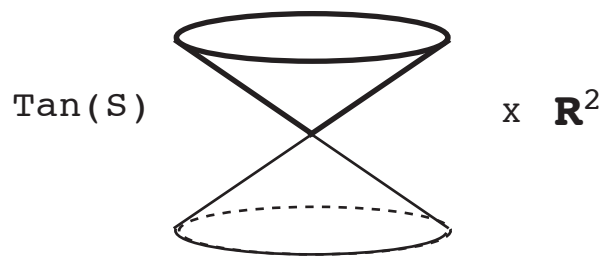

Figure 6: Tangent variety of Veronese surface.

See Figure 6. The tangent variety $\operatorname{Tan}(S)$ is not frontal. Note that, even if $S$ is algebraic, $\operatorname{Tan}(S)$ is semi-algebraic in general over the real numbers. For a generic surface $S \in \mathbf{R} P^{5}$, tangent varieties $\operatorname{Tan}(S)$ are perturbed into a non-frontal hypersurface.

Therefore the tangent variety $\operatorname{Tan}(S)$ to a generic surface $S \subset \mathbf{R} P^{5}$ is never frontal.

Let $V$ be a $(N+3)$-dimensional vector space. Let us consider a flag manifold

$$
\mathcal{F}=\mathcal{F}_{1,3}(V):=\left\{V_{1} \subset V_{3} \subset V\right\} \cong \operatorname{Gr}(2, T(P(V))),
$$

$\mathcal{F}_{1,3}(V)=3 N+2$, with local coordinates $x_{1}, x_{2}, y_{1}, \ldots, y_{N}, p_{1}, \ldots, p_{N}, q_{1}, \ldots, q_{N}$. The canonical differential system $\mathcal{T}=\mathcal{C}=\mathcal{C}_{1,3}$ is given by $d y_{i}=p_{i} d x_{1}+q_{i} d x_{2}, \quad(1 \leq i \leq N)$. A frontal mapgerm $f:\left(\mathbf{R}^{2}, 0\right) \rightarrow P(V)=\mathbf{R} P^{N+2}$ lifts to a $\mathcal{C}_{1,3}$-integral map-germs, therefore $f$ is an openings of $g=\left(x_{1} \circ f, x_{2} \circ f\right):\left(\mathbf{R}^{2}, 0\right) \rightarrow \mathbf{R}^{2}$ with the dense set of regular points.

Thus it is possible to study the singularities of tangent varieties to frontal surfaces can be studied as the singularity theory on $\mathcal{C}_{1,3}$-integral mappings. The general studies from this viewpoint are left to a forthcoming paper.

Now, let us consider another type of flag manifold: $\mathcal{F}_{1,3,5}(V)=\left\{V_{1} \subset V_{3} \subset V_{5} \subset V\right\}$. and the canonical system $\mathcal{N}=\mathcal{C}_{1,3,5} \subset T\left(\mathcal{F}_{1,3,5}(V)\right)$ defined by

$$
v \in \mathcal{C}_{1,3,5}\left(V_{1}, V_{3}, V_{5}\right) \Longleftrightarrow \pi_{i *}(v) \in T\left(\operatorname{Gr}\left(i, V_{i+2}\right)\right)\left(\subset T\left(\operatorname{Gr}\left(i, \mathbf{R}^{6}\right)\right), i=1,3 .\right.
$$

If $N=3$, then $\operatorname{dim}\left(\mathcal{F}_{1,3,5}\left(\mathbf{R}^{6}\right)\right)=13$ and $\operatorname{rank}\left(\mathcal{C}_{1,3,5}\right)=8$. In fact, $\mathcal{N}$ is given by

$$
\left\{\begin{aligned}
d x_{3}{ }^{0} & =x_{3}{ }^{1} d x_{1}{ }^{0}+x_{3}{ }^{2} d x_{2}{ }^{0} \\
d x_{4}{ }^{0} & =x_{4}{ }^{1} d x_{1}{ }^{0}+x_{4}{ }^{2} d x_{2}{ }^{0} \\
d x_{5}{ }^{0} & =x_{5}{ }^{1} d x_{1}{ }^{0}+x_{5}{ }^{2} d x_{2}{ }^{0} \\
d x_{5}{ }^{1} & =x_{5}{ }^{3} d x_{3}{ }^{1}+x_{5}{ }^{4} d x_{4}{ }^{1} \\
d x_{5}{ }^{2} & =x_{5}{ }^{3} d x_{3}{ }^{2}+x_{5}{ }^{4} d x_{4}{ }^{2}
\end{aligned}\right.
$$

for a system of projective local coordinates

$$
x_{1}^{0}, x_{2}{ }^{0}, x_{3}{ }^{0}, x_{4}{ }^{0}, x_{5}{ }^{0}, x_{3}{ }^{1}, x_{4}{ }^{1}, x_{5}{ }^{1}, x_{3}{ }^{2}, x_{4}{ }^{2}, x_{5}{ }^{2}, x_{5}{ }^{3}, x_{5}^{4}
$$

of $\mathcal{F}_{1,3,5}\left(V^{6}\right)$.

Proposition 9.2 Let $f:\left(\mathbf{R}^{2}, 0\right) \rightarrow P\left(V^{N+3}\right)$ be a frontal map-germ. Suppose that the regular locus of the tangent map $\operatorname{Tan}(f):\left(\mathbf{R}^{4}, 0\right) \rightarrow P(V)$ is dense. Then $\operatorname{Tan}(f)$ is frontal if and only if $f$ is the projection of a $\mathcal{C}_{1,3,5}$-integral map by $\pi_{1}: \mathcal{F}_{1,3,5}(V) \rightarrow P(V)$.

Proof: Suppose $\operatorname{Tan}(f)$ is frontal and $g:\left(\mathbf{R}^{4}, 0\right) \rightarrow \operatorname{Gr}(4, T(P(V)))=\mathcal{F}_{1,5}(V)$ is the Grassmannian lifting of $\operatorname{Tan}(f)$. Then $\left.g\right|_{\mathbf{R}^{2} \times 0}$ lifts a $\mathcal{C}_{1,3,5}$-integral map $F:\left(\mathbf{R}^{2}, 0\right) \rightarrow \mathcal{F}_{1,3,5}(V)$ and $\pi_{1} \circ F=f$. Conversely if $\pi_{1} \circ F=f$ for a $\mathcal{C}_{1,3,5}$-integral map $F$, then $\operatorname{Tan}(f)$ lifts to $G:\left(\mathbf{R}^{4}, 0\right) \rightarrow \mathcal{F}_{1,3,5}(V)$ by $G\left(s_{1}, s_{2}, t_{1}, t_{2}\right)=F\left(0,0, t_{1}, t_{2}\right)$. 
Let $V^{6}$ be a symplectic vector space. Let us consider the canonical contact structure on $P(V)=$ $\mathbf{R} P^{5}$. Let $S \subset \mathbf{R} P^{5}$ be a Legendre surface. Then $S$ lifts to a $\mathcal{C}_{1,3,5}$-integral surface. Therefore, by Theorem 9.2 , we have:

Corollary 9.3 Let $i:\left(\mathbf{R}^{2}, 0\right) \rightarrow \mathbf{R} P^{5}$ be a Legendre immersion-germ. Suppose the regular locus $\operatorname{Reg}(\operatorname{Tan}(i))$ of the tangent variety is dense in $\left(\mathbf{R}^{2}, 0\right)$. Then the tangent variety $\operatorname{Tan}(i):\left(\mathbf{R}^{2}, 0\right) \rightarrow$ $\mathbf{R} P^{5}$ is a frontal.

Definition 9.4 A point $p$ of a Legendre surface $S$ in $\mathbf{R} P^{5}$ is called an ordinary point if there exists a local projective-contact coordinates $x_{1}, x_{2}, x_{3}, x_{4}, x_{5}$ and a $C^{\infty}$ local coordinates $(u, v)$ of $S$ centred $p$ such that locally $S$ is given by

$$
\left\{\begin{array}{l}
x_{1}=u \\
x_{2}=v \\
x_{3}=\frac{1}{2} a u^{2}+b u v+\frac{1}{2} c v^{2}+\text { higher order terms } \\
x_{4}=\frac{1}{2} b u^{2}+c u v+\frac{1}{2} e v^{2}+\text { higher order terms } \\
x_{5}=-\left(\frac{1}{6} a u^{3}+\frac{1}{2} b u^{2} v+\frac{1}{2} c u v^{2}+\frac{1}{6} e v^{3}\right)+\text { higher order terms }
\end{array}\right.
$$

with

$$
\mathcal{D}=\left\{d x_{5}-x_{1} d x_{3}-x_{2} d x_{4}+x_{3} d x_{1}+x_{4} d x_{2}=0\right\}
$$

and

$$
\operatorname{rank}\left(\begin{array}{ccc}
a & b & c \\
b & c & e
\end{array}\right)=2 .
$$

An ordinary point $p$ is called hyperbolic (resp. elliptic, parabolic), if moreover

$$
H:=4\left(a c-b^{2}\right)\left(b e-c^{2}\right)-(a e-b c)^{2}
$$

is negative (resp. positive, zero).

Note that the set of hyperbolic (resp. elliptic) ordinary points is an open subset in $S$. Then we have the following fundamental result:

Theorem 9.5 The tangent variety $\operatorname{Tan}(S)$ to a Legendre surface $S$ in $\mathbf{R} P^{5}$ at a hyperbolic ordinary point (resp. an elliptic ordinary point) is locally diffeomorphic to $\left(D_{4}^{+}\right.$-singularity in $\left.\mathbf{R}^{3}\right) \times \mathbf{R}^{2}$ (resp. $\left(D_{4}^{-}\right.$-singularity in $\left.\left.\mathbf{R}^{3}\right) \times \mathbf{R}^{2}\right)$ in $\mathbf{R}^{5}$.

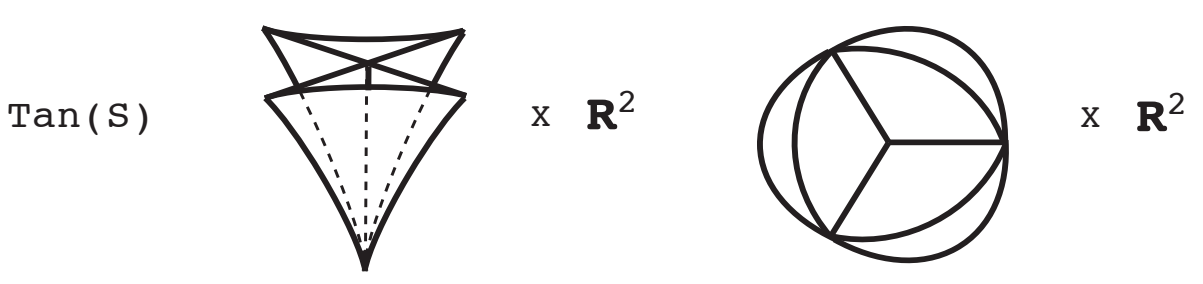

Figure 7: Tangent varieties along hyperbolic and elliptic ordinary points on a surface in $\mathbf{R} P^{5}$.

In [37], a simple criterion on $D_{4}$ has been found by Saji. The $D_{4}^{ \pm}$-singularity in $\mathbf{R}^{3}$ is given by the map-germ $\left(\mathbf{R}^{2}, 0\right) \rightarrow\left(\mathbf{R}^{3}, 0\right)$

$$
(u, v) \mapsto\left(u v, u^{2} \pm 3 v^{2}, u^{2} v \pm v^{3}\right)
$$


Theorem 9.6 ([37]) Let $f:\left(\mathbf{R}^{2}, 0\right) \rightarrow\left(\mathbf{R}^{3}, 0\right)$ be a front and $(f, \nu):\left(\mathbf{R}^{2}, 0\right) \rightarrow \mathbf{R}^{3} \times S^{2}$ a Legendre lift of $f$. Then $f$ is diffeomorphic to $D_{4}^{+}$(resp. $\left.D_{4}^{-}\right)$if and only if $f$ is of rank zero at 0 and the Hessian determinant of

$$
\lambda(u, v):=\operatorname{det}\left(\frac{\partial f}{\partial u}(u, v), \frac{\partial f}{\partial v}(u, v), \nu(u, v)\right)
$$

at $(0,0)$ is negative (resp. positive).

Note that $D_{4}$-singularity is not a generic singularity of wave-fronts in $\mathbf{R}^{3}$, but is a generic singularity of wave-fronts in $\mathbf{R}^{4}$. The criterion for $D_{4}$-singularities in $\mathbf{R}^{4}$ is also given in [37]. Moreover we remark that Saji's criterion is valid also for the case with parameters and it characterises the trivial deformation of $D_{4}$-singularity. In fact the same line of proof in [37] works as well for the case with parameters:

Theorem 9.7 Let $F=\left(f_{t}\right)_{t \in\left(\mathbf{R}^{r}, 0\right)}:\left(\mathbf{R}^{2} \times \mathbf{R}^{r}, 0\right) \rightarrow\left(\mathbf{R}^{3}, 0\right)$ be a family of fronts and $(F, N)=$ $\left(f_{t}, \nu_{t}\right):\left(\mathbf{R}^{2} \times \mathbf{R}^{r}, 0\right) \rightarrow \mathbf{R}^{3} \times S^{2}$ a family of Legendre lifts of $F$. Then $F$ is diffeomorphic to the trivial deformation of $D_{4}^{+}$(resp. $\left.D_{4}^{-}\right)$if and only if $f_{t}$ is of rank zero at 0 and the Hessian determinant of

$$
\lambda(u, v, t):=\operatorname{det}\left(\frac{\partial f_{t}}{\partial u}(u, v), \frac{\partial f_{t}}{\partial v}(u, v), \nu_{t}(u, v)\right)
$$

with respect to $(u, v)$ at $(0,0, t)$ is negative (resp. positive), for any $t \in\left(\mathbf{R}^{r}, 0\right)$.

Proof of Theorem 9.5: Let $x_{1}=u, x_{2}=v$,

$$
\begin{aligned}
& x_{3}=\frac{1}{2} a u^{2}+b u v+\frac{1}{2} c v^{2}+\varphi(u, v), \\
& x_{4}=\frac{1}{2} b u^{2}+c u v+\frac{1}{2} e v^{2}+\psi(u, v), \\
& x_{5}=-\left(\frac{1}{6} a u^{3}+\frac{1}{2} b u^{2} v+\frac{1}{2} c u v^{2}+\frac{1}{6} e v^{3}\right)+\rho(u, v),
\end{aligned}
$$

$\operatorname{ord}(\varphi) \geq 3, \operatorname{ord}(\psi) \geq 3$, and

$$
\rho_{u}=u \varphi_{u}+v \psi_{u}-\varphi, \quad \rho_{u}=u \varphi_{u}+v \psi_{u}-\psi
$$

As an integrability condition, we have that $\varphi_{v}=\psi_{u}$. The tangent map of $S$ is given by $x_{1}=$ $u+s, x_{2}=v+t$

$$
\begin{aligned}
x_{3}= & \frac{1}{2} a u^{2}+b u v+\frac{1}{2} c v^{2}+\varphi+s\left(a u+b v+\varphi_{u}\right)+t\left(b u+c v+\varphi_{v}\right), \\
x_{4}= & \frac{1}{2} b u^{2}+c u v+\frac{1}{2} e v^{2}+\psi+s\left(b u+c v+\psi_{u}\right)+t\left(c u+e v+\psi_{v}\right), \\
x_{5}= & -\left(\frac{1}{6} a u^{3}+\frac{1}{2} b u^{2} v+\frac{1}{2} c u v^{2}+\frac{1}{6} e v^{3}\right)+\rho \\
& \quad+s\left(-\frac{1}{2} a u^{2}-b u v-\frac{1}{2} c v^{2}+\rho_{u}\right)+t\left(-\frac{1}{2} b u^{2}-c u v-\frac{1}{2} e v^{2}+\rho_{v}\right) .
\end{aligned}
$$

Take the transversal slice $s=-u, t=-v$. Then we have map-germ $g:\left(\mathbf{R}^{2}, 0\right) \rightarrow\left(\mathbf{R}^{3}, 0\right)$,

$$
\begin{aligned}
& g_{1}(u, v)=-\frac{1}{2} a u^{2}-b u v-\frac{1}{2} c v^{2}+\varphi-u \varphi_{u}-v \varphi_{v}, \\
& g_{2}(u, v)=-\frac{1}{2} b u^{2}-c u v-\frac{1}{2} e v^{2}+\psi-u \psi_{u}-v \psi_{v}, \\
& g_{3}(u, v)=\frac{1}{3} a u^{3}+b u^{2} v+c u v^{2}+\frac{1}{3} e v^{3}+\rho-u \rho_{u}-v \rho_{v} .
\end{aligned}
$$

We show that $g$ is diffeomorphic to $D_{4}$-singularity, by using Saji's criterion (Theorem 9.6). 
First, we have $d g_{3}=-u d g_{1}-v d g_{2}$. Therefore $g$ is a front and we can take $\nu=\frac{1}{\sqrt{u^{2}+v^{2}+1}}(u, v, 1)$. Second, we see $f$ is of rank zero. Third,

$$
\begin{aligned}
\lambda(u, v) & =\operatorname{det}\left(g_{u}, g_{v}, \nu\right)=\operatorname{det}\left(\begin{array}{ccc}
g_{1 u} & g_{1 v} & u \\
g_{2 u} & g_{2 v} & v \\
0 & 0 & \sqrt{u^{2}+v^{2}+1}
\end{array}\right) \\
& =\sqrt{u^{2}+v^{2}+1}\left(g_{1 u} g_{2 v}-g_{1 v} g_{2 u}\right)
\end{aligned}
$$

The 2-jet of $h:=g_{1 u} g_{2 v}-g_{1 v} g_{2 u}$ at 0 is given by

$$
j^{2} h(0)=\left(a c-b^{2}\right) u^{2}+(a e-b c) u v+\left(b e-c^{2}\right) v^{2} \quad\left(\bmod . \mathfrak{m}_{2}^{3}\right) .
$$

Therefore we have that the Hessian determinant of $\lambda$ at 0 is given by

$$
H=\operatorname{det}\left(\begin{array}{cc}
2\left(a c-b^{2}\right) & a e-b c \\
a e-b c & 2\left(b e-c^{2}\right)
\end{array}\right)
$$

By Theorem 9.6, we see that $g$ is diffeomorphic to $D_{4}^{ \pm}$if and only if $\mp H>0$. Moreover, we can show similarly that, regarding $S$ as the parameter space, the tangent map-germ is diffeomorphic to the trivial unfolding of $D_{4}$-singularity with two parameters, by using Theorem 9.7. Hence we have Theorem 9.5 ,

\section{Tangent maps to frontal maps and open problems.}

Let $V$ be a $(N+2 n)$-dimensional vector space with positive natural numbers $N, n$. Consider the flag manifolds:

$$
\mathcal{F}_{1, n+1,2 n+1}=\mathcal{F}_{1, n+1,2 n+1}(V):=\left\{V_{1} \subset V_{n+1} \subset V_{2 n+1} \subset V\right\},
$$

with the canonical differential system $\mathcal{C}_{1, n+1,2 n+1}$, and

$$
\mathcal{F}_{1, n+1}=\mathcal{F}_{1, n+1}(V):=\left\{V_{1} \subset V_{n+1} \subset V\right\},
$$

with the canonical differential system $\mathcal{C}_{1, n+1}$. Note that $\mathcal{F}_{1, n+1}$ is identified with the Grassmannian bundle $\operatorname{Gr}(n, T(P(V)))$. Consider the canonical projections

$$
\mathcal{F}_{1, n+1,2 n+1} \stackrel{\Pi}{\longrightarrow} \mathcal{F}_{1, n+1} \stackrel{\pi}{\longrightarrow} \mathcal{F}_{1}=P(V)=\mathbf{R} P^{N+2 n-1} .
$$

Similarly to the proof of Proposition 9.2, we have

Proposition 10.1 Let $f:\left(\mathbf{R}^{n}, 0\right) \rightarrow \mathbf{R} P^{N+2 n-1}$ be a frontal map-germ. Suppose the regular locus $\operatorname{Reg}(\operatorname{Tan}(f))$ of the tangent map $\operatorname{Tan}(f):\left(\mathbf{R}^{2 n}, 0\right) \rightarrow \mathbf{R} P^{N+2 n-1}$ is dense in $\left(\mathbf{R}^{2 n}, 0\right)$. Then $\operatorname{Tan}(f)$ is frontal if and only if the Grassmannian lift $\tilde{f}:\left(\mathbf{R}^{n}, 0\right) \rightarrow \mathcal{F}_{1, n+1}$ of $f$ for $\pi$, lifts to a

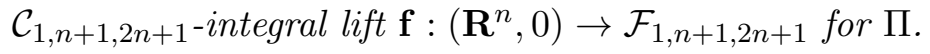

It is natural to proceed to consider the tangent varieties to Legendre submanifolds.

Let $V$ be a $(2 n+2)$-dimensional symplectic vector space. Consider the Lagrange (isotropic) flag manifold:

$$
\mathcal{F}_{\text {Lag }}=\mathcal{F}_{\text {Lag }}(V):=\left\{V_{1} \subset V_{n+1} \subset V \mid V_{n+1} \text { is Lagrange. }\right\},
$$

with the canonical differential system $\mathcal{E} \subset T \mathcal{F}_{\text {Lag. }}$. In general we have

Corollary 10.2 Let $g:\left(\mathbf{R}^{n}, 0\right) \rightarrow \mathcal{F}_{\text {Lag }}$ be $\mathcal{E}$-integral and $\operatorname{Tan}\left(\pi_{1} \circ g\right):\left(\mathbf{R}^{2 n}, 0\right) \rightarrow P(V)$ the tangent map-germ of $\pi_{1} \circ g:\left(\mathbf{R}^{n}, 0\right) \rightarrow P(V)$. Suppose that $\operatorname{Reg}\left(\operatorname{Tan}\left(\pi_{1} \circ g\right)\right)$ is dense in $\left(\mathbf{R}^{n}, 0\right)$. Then $\operatorname{Tan}\left(\pi_{1} \circ g\right)$ is frontal. 
Proof: Note that $\mathcal{F}_{\text {Lag }}$ is embedded in $\mathcal{F}_{1, n+1,2 n+1}$ by $\left(V_{1}, V_{n+1}\right) \mapsto\left(V_{1}, V_{n+1}, V_{1}^{s}\right)$, where $V_{1}^{s}$ is the symplectic skew-orthogonal to $V_{1}$, and $\mathcal{E}$ is the restriction of $\mathcal{C}_{1, n+1,2 n+1}$. Therefore Proposition 10.2 follows from Proposition 10.1 .

Here we give alternative direct proof. Since $f$ is Legendre, $f=(\lambda, \mu, \nu)$ satisfies $d \mu=$ $\sum_{i=1}^{n}\left(\nu_{i} d \lambda_{i}-\lambda_{i} d \nu_{i}\right)$. The tangent map-germ $\operatorname{Tan}(f)=(\Lambda, M, N)$ is given by

$$
\left(\begin{array}{c}
\Lambda \\
M \\
N
\end{array}\right)=\left(\begin{array}{c}
\lambda \\
\mu \\
\nu
\end{array}\right)+\sum_{j=1}^{n} s_{j}\left(\begin{array}{c}
\partial \lambda / \partial u_{j} \\
\partial \mu / \partial u_{j} \\
\partial \nu / \partial u_{j}
\end{array}\right) .
$$

Then we have

$$
\begin{aligned}
d M= & d \mu+\sum_{j=1}^{n} s_{j} d\left(\partial \mu / \partial u_{j}\right)+\sum_{j=1}^{n}\left(\partial \mu / \partial u_{j}\right) d s_{j} \\
= & d \mu+\sum_{i=1}^{n} \sum_{j=1}^{n} s_{j}\left(\nu_{i} d\left(\partial \lambda_{i} / \partial u_{j}\right)-\lambda_{i} d\left(\partial \nu_{i} / \partial u_{j}\right)\right) \\
& \quad+\sum_{i=1}^{n} \sum_{j=1}^{n}\left(\nu_{i}\left(\partial \lambda_{i} / \partial u_{j}\right)-\lambda_{i}\left(\partial \nu_{i} / \partial u_{j}\right)\right) d s_{j} \\
= & \sum_{i=1}^{n}\left(\nu_{i} d \Lambda_{i}-\lambda_{i} d N_{i}\right) .
\end{aligned}
$$

Thus $M \in \mathcal{R}_{(\Lambda, N)}$ and $\operatorname{Tan}(f)$ is frontal.

Then Corollary 10.2 implies

Corollary 10.3 Let $f:\left(\mathbf{R}^{n}, 0\right) \rightarrow P(V)=\mathbf{R} P^{2 n+1}$ be a germ of Legendre immersion and $\operatorname{Tan}(f)$ : $\left(\mathbf{R}^{2 n}, 0\right) \rightarrow P(V)$ the tangent map-germ of $f$. Suppose that $\operatorname{Reg}(\operatorname{Tan}(f))$ is dense in $\left(\mathbf{R}^{n}, 0\right)$. Then $\operatorname{Tan}(f)$ is frontal.

We conclude the paper by posing open generic classification problems, which remain to be solved first:

Problem 1: Classify the singularities of tangent varieties to generic contact-integral curves in $P\left(V^{2 n+2}\right) \cong \mathbf{R} P^{2 n+1}$ for a symplectic vector space $V$ of dimension $2 n+2$, under diffeomorphisms and contactomorphisms.

Problem 2: Classify the singularities of tangent varieties to generic surfaces in $\mathbf{R} P^{5}$. It would be natural to relate singularities of tangent variety to the method of height function or hight family (cf. [39] [31]).

Problem 3: Classify the singularities of tangent varieties to generic frontal surfaces (projections of generic $\mathcal{C}_{1,3}$-integral surfaces in $\left.\mathcal{F}_{1,3}\left(\mathbf{R}^{6}\right)\right)$ in $\mathbf{R} P^{5}$.

Problem 4: Classify the singularities of tangent varieties to projections in $\mathbf{R} P^{5}$ of generic $\mathcal{C}_{1,3,5^{-}}$ integral surfaces in $\mathcal{F}_{1,3,5}\left(\mathbf{R}^{6}\right)$.

Problem 5: Classify the singularities of tangent varieties to Legendre surfaces in $\mathbf{R} P^{5}$ along parabolic ordinary points. Moreover classify the singularities of tangent varieties of generic Legendre surfaces in $\mathbf{R} P^{5}$. (See $₫ 9$ )

\section{References}

[1] M.A. Akivis, V.V. Goldberg, Differential geometry of varieties with degenerate Gauss maps, CMS Books in Mathematics, 18, Springer-Verlag, New York, (2004).

[2] V.I. Arnol'd, Lagrangian manifolds with singularities, asymptotic rays, and the open swallowtail, Funkt. Anal. i Prilozhen., 15-4 (1981), 1-14. English transl. Funct. Anal. and its Appl., 15-4 (1981), 235-246.

[3] V.I. Arnol'd, Singularities of caustics and wave fronts, Math. its Appl. (Soviet Series), 62. Kluwer Academic Publishers, (1990). 
[4] V.I. Arnol'd, Catastrophe theory, 3rd edition, Springer-Verlag, (1992).

[5] T. Bröcker, Differentiable Germs and Catastrophes, London Math. Soc. Lecture Note Series 17, Cambridge Univ. Press (1975).

[6] A. Cayley, On certain developable surfaces, Quarterly J. Pure Appl. Math., 6 (1864), 108-126.

[7] S. Chino, S. Izumiya, Lightlike developables in Minkowski 3-space, Demonstratio Math., 43-2 (2010), 387-399.

[8] J.P. Cleave, The form of the tangent-developable at points of zero torsion on space curves, Math. Proc. Camb. Philos. Soc., 88-3 (1980), 403-407.

[9] G. Fischer, J. Piontkowski, Ruled varieties, An introduction to algebraic differential geometry, Advanced Lectures in Math., Friedr. Vieweg \& Sohn, (2001).

[10] G. Fischer, H. Wu, Developable complex analytic submanifolds, Intern. J. Math., 6-2 (1995), $229-272$.

[11] W. Fulton, R. Lazarsfeld, Connectivity and its applications in algebraic geometry, Lecture Notes in Math., 862, Springer, (1981), pp. 26-92.

[12] J.W. Bruce, P.J. Giblin, Curves and singularities, A geometrical introduction to singularity theory, 2nd ed., Cambridge Univ. Press, (1992).

[13] A.B. Givental', Whitney singularities of solutions of partial differential equations, J. Geom. Phys. 15-4 (1995) 353-368.

[14] S. Ghosh, J.H. Rieger, Singularities of secant maps of immersed surfaces, Geom. Dedicata 121 (2006) 73-87.

[15] G. Ishikawa, Y. Machida, M. Takahashi, Asymmetry in singularities of tangent surfaces in contact-cone Legendrenull duality, Journal of Singularities, 3 (2011), 126-143.

[16] G. Ishikawa, Parametrization of a singular Lagrangian variety, Trans. Amer. Math. Soc., 331-2 (1992), 787798.

[17] G. Ishikawa, Determinacy of the envelope of the osculating hyperplanes to a curve, Bull. London Math. Soc., 25 (1993), 603-610.

[18] G. Ishikawa, Parametrized Legendre and Lagrange varieties, Kodai Math. J., 17-3 (1994), $442-451$.

[19] G. Ishikawa, Developable of a curve and determinacy relative to osculation-type, Quart. J. Math. Oxford, 46 (1995).

[20] G. Ishikawa, Symplectic and Lagrange stabilities of open Whitney umbrellas, Invent. math., bf 126 (1996), 215-234.

[21] G. Ishikawa, Generic bifurcations of framed curves in a space form and their envelopes, Topology and its Appl., 159 (2012), 492-500.

[22] G. Ishikawa, S. Janeczko, Symplectic bifurcations of plane curves and isotropic liftings, Quarterly J. Math. Oxford, 54 (2003), 73-102.

[23] T.A. Ivey and J.M. Landsberg, Cartan for beginners: differential geometry via moving frames and exterior differential systems, Amer. Math. Soc. (2003).

[24] S. Izumiya, H. Katsumi, T. Yamasaki, The rectifying developable and the spherical Darboux image of a space curve, Causticsf98, Banach Center Publ., 50 (1999), 137-149.

[25] S. Izumiya, K. Saji, The mandala of Legendrian dualities for pseudo-spheres in Lorenz-Minkowski space and "flat" spacelike surfaces. Journal of Singularities 2 (2010), 92-127.

[26] M. Kossowski, Prescribing invariants for integral surfaces in the Grassmann bundle of 2-planes in 4-space, Topology 35-1 (1996), 1-12.

[27] M. Kossowski, Realizing a singular first fundamental form as a nonimmersed surface in Euclidean 3-space, J. Geom. 81 (2004), 101-113.

[28] M. Kossowski, M. Scherfner, Total curvature for $C^{\infty}$-singular surfaces with limiting tangent bundle, Ann. Global Anal. Geom., 28-2 (2005), 179-199.

[29] J.M. Landsberg, J. Weyman, On tangential varieties of rational homogeneous varieties, J. London Math. Soc., 76-2 (2007), 513-530.

[30] J. Martinet, Singularities of Smooth Functions and Maps, London Math. Soc. Lecture Note Series 58, Cambridge Univ. Press (1982).

[31] D.K.H. Mochida, M.C. Romero-Fuster, M.A.S. Ruas, Inflection points and nonsingular embeddings of surfaces in $\mathbf{R}^{5}$, Rockey Mount. J. Math., 33-3 (2003) 995-1009.

[32] D. Mond, On the tangent developable of a space curve, Math. Proc. Cambridge Philos. Soc., 91-3 (1982), 351-355. 
[33] D. Mond, Singularities of the tangent developable surface of a space curve, Quart. J. Math. Oxford, 40-157 (1989), 79-91.

[34] D. Mond, Deformations which preserve the non-immersive locus of a map-germ, Math. Scand., 66 (1990), 21-32.

[35] I.R. Porteous, Geometric Differentiation: For the intelligence of curves and surfaces, Camb. Univ. Press., (1994).

[36] K. Saji, M. Umehara, K. Yamada, The geometry of fronts, Annals of Math., 169 (2009), 491-520.

[37] K. Saji, Criteria for $D_{4}$ singularities of wave fronts, Tohoku Math. J., 63 (2011), 137-147.

[38] O.P. Scherbak, Projective dual space curves and Legendre singularities, Trudy Tbiliss Univ., 232-233 (1982), 280-336.

[39] C.T.C. Wall, Geometric properties of generic differentiable manifolds, Lecture Notes in Math., 597, Springer, (1977), pp. 707-774.

[40] C.T.C. Wall, Plücker formulae for singular space curves, Math. Proc. Camb. Phil. Soc. 151 (2011), $129-143$.

[41] F.L. Zak, Tangents and Secants of Algebraic Varieties, Transl. of Math. Monographs 127, Amer. Math. Soc., (1993). 